REPRESENTATION THEORY

An Electronic Journal of the American Mathematical Society

Volume 10, Pages 21-47 (January 9, 2006)

S $1088-4165(06) 00279-2$

\title{
HOWE QUOTIENTS OF UNITARY CHARACTERS AND UNITARY LOWEST WEIGHT MODULES
}

\author{
HUNG YEAN LOKE, WITH AN APPENDIX BY SOO TECK LEE
}

\begin{abstract}
In this paper, let $\left(G, G^{\prime}\right)$ be the dual pair $(\widetilde{\mathrm{Sp}}(p, \mathbb{R}), \tilde{\mathrm{O}}(n, m))$. We will determine the composition series of the Howe quotients of $G^{\prime}$ which are lifts from one-dimensional unitary representations of $G$ and unitary lowest weight modules of $G$. We will also determine the unitarizability of the subquotients. Our method also works for the dual pairs $(\widetilde{\mathrm{U}}(p, q), \widetilde{\mathrm{U}}(n, m))$ and $\left(\tilde{\mathrm{O}}^{*}(2 p), \widetilde{\mathrm{Sp}}(n, m)\right)$.
\end{abstract}

\section{INTRODUCTION}

1.1. Throughout this paper, $\operatorname{Sp}(N)$ will denote the compact Lie group of type $C_{N}$ and $\operatorname{Sp}(N, \mathbb{R})$ will denote its split form. In this paper we consider the dual pair $(\widetilde{\mathrm{Sp}}(p, \mathbb{R}), \tilde{\mathrm{O}}(n, m))$ in $\widetilde{\mathrm{Sp}}(p(n+m), \mathbb{R})$. Here the tilde above each group denotes a two-fold central extension which may be trivial. See $\$ 2.2$ for the definitions of the extensions. We will determine the composition series and the unitarizability of the (maximal) Howe quotients which are lifts to $\tilde{O}(n, m)$ from following genuine unitary representations of $\widetilde{\operatorname{Sp}}(p, \mathbb{R})$ :

(i) One-dimensional genuine unitary representations and,

(ii) all genuine unitary lowest weight modules.

The Howe quotient lifts from one-dimensional unitary characters are $K$-multiplicity free but the lifts from the unitary lowest weight modules are not $K$-multiplicity free. See $\$ 2.3$ for the definitions of genuine representations and Howe quotients. By [Ho2] it has a unique quotient called the local theta lift. Appendix A describes the Howe quotients which are lifts from the one-dimensional genuine representation for the dual pairs $(\widetilde{\mathrm{Sp}}(p, \mathbb{R}), \tilde{\mathrm{O}}(n, n))$.

We remark that our method also works for the dual pairs $(\widetilde{\mathrm{U}}(p, q), \widetilde{\mathrm{U}}(n, m))$ and $\left(\tilde{\mathrm{O}}^{*}(2 p), \widetilde{\mathrm{Sp}}(n, m)\right)$.

1.2. Let $\xi_{\mathrm{s}}$ denote the (unique) genuine unitary one-dimensional representation of $\widetilde{\mathrm{Sp}}(p, \mathbb{R})$ and let $\Omega(1)$ or $\Omega_{p}^{n, m}(1)$ denote its Howe quotient lift to $\tilde{\mathrm{O}}(n, m)$. We will see in 2.4 that $\xi_{\mathrm{s}}$ exists if and only if $n+m$ is even. The Howe quotient $\Omega(1)$ is a

Received by the editors March 8, 2005 and, in revised form, September 13, 2005.

2000 Mathematics Subject Classification. Primary 22E46, $22 \mathrm{E} 47$.

Key words and phrases. Howe quotients, local theta lifts, unitary characters, unitary lowest weight modules, composition series.

The research of Hung Yean Loke was partially funded by the NUS Academic Research Grant R-146-000-026-112.

The research of Soo Teck Lee was partially funded by the NUS Academic Research Grant R-146-000-026-112. 
Harish-Chandra module of $\tilde{\mathrm{O}}(n, m)$. Let $K_{n, m}=\tilde{\mathrm{O}}(n) \times{ }_{2} \tilde{\mathrm{O}}(m)$ denote the maximal compact subgroup of $\tilde{\mathrm{O}}(n, m)$. Here $\tilde{\mathrm{O}}(n) \times_{2} \tilde{\mathrm{O}}(m)$ denotes $(\tilde{\mathrm{O}}(n) \times \tilde{\mathrm{O}}(m)) /(\mathbb{Z} / 2 \mathbb{Z})$. We will compute the $K_{n, m}$-types of $\Omega(1)$ in $\$ 3.5$ and we will see that the $K_{n, m}$-types have two distinctive properties: First it is $K_{n, m}$-multiplicity free and second it is $\tilde{\mathrm{O}}(n) \times 1$-admissible. These two properties are vital to our investigations.

Our first main result gives the composition series of $\Omega(1)$ and the unitarizability of its irreducible subquotients. By symmetry we will assume that $n \leq m$.

Theorem 1.2.1. Suppose the dual pair is $(\widetilde{\mathrm{Sp}}(p, \mathbb{R}), \tilde{\mathrm{O}}(n, m))$ where $n \leq m$, then $\Omega_{p}^{n, m}(1) \neq 0$ iff one of the following situations holds:

(I) $2 p \leq n \leq m$ and $m \equiv n(\bmod 2)$. We exclude the case $2 p=n=m$.

(II) $n=m \leq 2 p$.

(III) $p \leq n \leq 2 p-1$ and $m=n+2$.

The Howe quotient $\Omega_{p}^{n, m}(1)$ is irreducible if and only if we are in Cases (I) and (III). In these two cases $\Omega_{p}^{n, m}(1)$ is unitarizable.

In Case (II) $\Omega_{p}^{n, n}(1)$ is reducible and its composition series is given in Appendix A and we thank Soo Teck Lee for allowing us to include it in this paper. Case (I) is a special case of a result of Zhu-Huang [ZH] and Li [Li1] (see \$2.5). Nevertheless, we will sketch a proof of it using the method in this paper.

1.3. Next we discuss the Howe quotients which are lifts of unitary lowest weight modules. The Lie group $\tilde{\mathrm{O}}(n, m)$ contains the Lie subgroup $\tilde{\mathrm{O}}(n, r) \cdot \tilde{\mathrm{O}}(m-r)$ where $r<m$. Under the compact correspondences of the dual pair $(\widetilde{\mathrm{Sp}}(p, \mathbb{R}), \tilde{\mathrm{O}}(m-r))$ [KV], every genuine unitary irreducible representation $\tau_{m-r}^{\mu}$ of $\tilde{\mathrm{O}}(m-r)$ corresponds to a genuine lowest weight module $\Theta(\mu)$ of $\widetilde{\mathrm{Sp}}(p, \mathbb{R})$. See $₫ 3.4$ for the definition of $\Theta(\mu)$.

Since $\Omega_{p}^{n, m}(1)$ is $\tilde{O}(n) \times 1$-admissible, it makes sense to consider $\Omega_{p}^{n, m}(1)$ as a Harish-Chandra module of $\tilde{\mathrm{O}}(n, r) \cdot \tilde{\mathrm{O}}(m-r)$. This is an example of discretely decomposable restriction in $\mathrm{Ko} 2$. Let $\Omega_{p}^{n, r}(1, \mu)$ or $\Omega(1, \mu)$ denote the Howe quotient lifted from the unitary lowest weight module $\Theta(\mu)$ of $\widetilde{\mathrm{Sp}}(p, \mathbb{R})$ to $\tilde{\mathrm{O}}(n, r)$. By Kulda's see-saw pair argument (see Lemma 4.2.1), $\Omega_{p}^{n, m}(1)$ decomposes as a direct sum of Harish-Chandra modules of $\tilde{\mathrm{O}}(n, r) \times \tilde{\mathrm{O}}(m-r)$,

$$
\Omega_{p}^{n, m}(1)=\sum_{\mu} \Omega_{p}^{n, r}(1, \mu) \otimes \tau_{m-r}^{\mu}
$$

where the sum is taken over all genuine finite-dimensional representations of $\tilde{\mathrm{O}}(m-r)$.

1.4. By (1), it is not surprising that the representations $\Omega_{p}^{n, m}(1)$ and $\Omega_{p}^{n, r}(1, \mu)$ are closely related. Let $v_{\mu}$ denote a highest weight vector of $\tau_{m-r}^{\mu}$. Let $\Omega(1)_{v_{\mu}}$ denote the subspace of $\Omega(1)$ which is a span of $h\left(v_{\mu}\right)$ for all $h \in \operatorname{Hom}_{\tilde{O}(m-r)}\left(\tau_{m-r}^{\mu}, \Omega_{p}^{n, r}(1)\right)$. By (11), we may regard $\Omega(1, \mu) \simeq \Omega(1, \mu) \otimes v_{\mu} \simeq \Omega(1)_{v_{\mu}}$ as a submodule of $\Omega(1)$.

Let $K_{n, r}:=\tilde{\mathrm{O}}(n) \times_{2} \tilde{\mathrm{O}}(r)$ denote the maximal compact subgroup of $\tilde{\mathrm{O}}(n, r)$. One can compute the $K_{n, r}$-types of $\Omega(1, \mu)$ from the $K_{n, m}$-types of $\Omega(1)$ by applying the branching rule $K_{n, m} \downarrow K_{n, r} \cdot \tilde{\mathrm{O}}(m-r)$ to (1). In particular, we can check whether $\Omega(1, \mu)$ is nonzero.

We remark that the process is reversible in the following sense. Let $\pi$ denote a Harish-Chandra module of a genuine irreducible unitary lowest weight module of 
$\widetilde{\mathrm{Sp}}(p, \mathbb{R})$. By [DER] and [EHW], there exists a compact $\tilde{\mathrm{O}}\left(r^{\prime}\right)$ and an irreducible genuine representation $\tau_{r^{\prime}}^{\mu^{\prime}}$ of $\tilde{\mathrm{O}}\left(r^{\prime}\right)$ with highest weight $\mu^{\prime}$ such that $\pi=\Theta\left(\mu^{\prime}\right)$ is the theta lift from $\tau_{r^{\prime}}^{\mu^{\prime}}$. Then (1) remains valid, namely, the Howe quotient lift $\Omega_{p}^{n, r}\left(1, \mu^{\prime}\right)$ of $\pi$ to $\tilde{\mathrm{O}}(n, r)$ is a submodule of the Howe quotient lift $\Omega_{p}^{n, r+r^{\prime}}(1)$ of the genuine one-dimensional character $\xi_{\mathrm{s}}$ from $\widetilde{\mathrm{Sp}}(p, \mathbb{R})$ to $\tilde{\mathrm{O}}\left(n, r+r^{\prime}\right)$. In particular, if $\Omega_{p}^{n, r+r^{\prime}}(1)=0$, then $\Omega_{p}^{n, r}\left(1, \mu^{\prime}\right)=0$.

1.5. Next we investigate the subquotients of $\Omega_{p}^{n, m}(1)$ and $\Omega_{p}^{n, r}(1, \mu)$ in (10). Suppose $W_{1} \subset W_{2}$ are $\left(\mathfrak{s o}(n, m), K_{n, m}\right)$-submodules of $\Omega_{p}^{n, m}(1)$, then $W_{i}^{\prime}:=W_{i} \cap$ $\Omega_{p}^{n, m}(1, \mu)(i=1,2)$ are admissible $\left(\mathfrak{s o}(n, r), K_{n, r}\right)$-submodules of $\Omega_{p}^{n, r}(1, \mu)$ and $W_{2}^{\prime} / W_{1}^{\prime}$ is a subquotient. In this way, subquotients $\tilde{\Omega}:=W_{2} / W_{1}$ of $\Omega(1)$ give rise to (possibly zero) subquotients $W_{2}^{\prime} / W_{1}^{\prime}$ of $\Omega(1, \mu)$. Before we state our first main result, we need two more notations. Let $\tilde{\Omega}_{v_{\mu}}$ denote the subspace of $\tilde{\Omega}$ where the compact $\tilde{\mathrm{O}}(m-r)$ acts by the highest weight $\mu$. Hence $\tilde{\Omega}_{v_{\mu}}$ is isomorphic to $W_{2}^{\prime} / W_{1}^{\prime}$. We refer to [Al] and [LL1] for the definition of the module diagram of a Harish-Chandra module of finite length.

Theorem 1.5.1. $\quad$ (i) Suppose $\tilde{\Omega}$ is a nonzero $\left(\mathfrak{s o}(n, m), K_{n, m}\right)$-subquotient of $\Omega(1)$. Then $\tilde{\Omega}$ is irreducible if and only if for every highest weight $\mu$ of $\tilde{\mathrm{O}}(m-r), \tilde{\Omega}_{v_{\mu}}$ is either zero or an admissible irreducible $\left(\mathfrak{s o}(n, r), K_{n, r}\right)$ subquotient of the Howe quotient $\Omega(1, \mu)$.

(ii) All irreducible subquotients of $\Omega(1, \mu)$ are of the form $\tilde{\Omega}_{v_{\mu}}$ given in (i).

(iii) The module diagram of $\Omega(1, \mu)$ is a spanning subgraph of $\Omega(1)$.

Similar statements about unitarizability hold.

Theorem 1.5.2. Suppose $\tilde{\Omega}$ is an irreducible $\left(\mathfrak{s o}(n, m), K_{n, m}\right)$-subquotient of $\Omega(1)$ as in Theorem 1.5.1(i). Then $\tilde{\Omega}$ is unitarizable if and only if for every highest weight $\mu$ of $\tilde{\mathrm{O}}(m-r), \tilde{\Omega}_{v_{\mu}}$ is either zero or a unitarizable irreducible $\left(\mathfrak{s o}(n, r), K_{n, r}\right)$ subquotient of the Howe quotient $\Omega(1, \mu)$.

Suppose $\Omega(1)$ belongs to Case (I) or (III) in Theorem 1.2.1, the above two the-

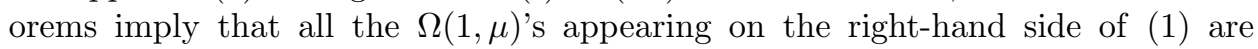
irreducible and unitarizable. See Corollary 4.2.2.

Using the above two theorems, we will describe the composition series of $\Omega_{p}^{n, r}(1, \mu)$ and the unitarizability of all its irreducible subquotients in $\$ 8.8$. We identify some of the unitarizable theta lifts in $\$ 9$ with Vogan-Zuckerman's $A_{\mathfrak{q}}(\lambda)$.

If we set $p=1$, then $\Omega_{1}^{n, m}(1)$ is a ladder representation of $\tilde{\mathrm{O}}(n, m)$ and our results overlap with Theorem $\mathrm{C}$ in $\mathrm{KoO}$.

1.6. We will briefly describe our method of proof. The $K_{n, m}$-types of $\Omega(1)$ is multiplicity free and this makes it possible to describe the Lie algebra action of $\mathfrak{s o}(n, m)$ on $\Omega(1)$ via a family of $K_{n, m}$-module homomorphisms between finitedimensional representations of $K_{n, m}$. These homomorphisms are called transition coefficients and we denote them by $T_{\lambda, \lambda^{\prime}}$ (see $\$ 5.2$ ). Likewise by (1) it is also possible to describe the Lie algebra action of $\mathfrak{s o}(n, r)$ on $\Omega(1, \mu)$ by its transition coefficients $t_{\lambda, \lambda^{\prime}}$. The transition coefficients $T_{\lambda, \lambda^{\prime}}$ and $t_{\lambda, \lambda^{\prime}}$ uniquely determine each other.

From here on, we need two approaches. For Case (II) we embed $\Omega(1)$ into a degenerate principal series representation of $\tilde{\mathrm{O}}(n, n)$ and we prove Theorems 1.5 .1 and 1.5 .2 using an idea in LL1. 
For Cases (I) and (III), we have to go back and forth between Theorems 1.2.1. 1.5 .1 and 1.5.2. The main step is to determine $\Omega_{p}^{n, 1}(1, \mu)$ in the special case when $\tilde{\mathrm{O}}(n, r)=\tilde{\mathrm{O}}(n, 1)$ in (11). By checking its $K_{n, 1}$-types and infinitesimal character against the list of irreducible representations of $\mathrm{SO}_{0}(n, 1)$ in [Hi] and [KG], we can deduce the Langlands parameters of $\Omega_{p}^{n, 1}(1, \mu)$. We will show that for every $\mu$, $\Omega(1, \mu)$ is an irreducible and unitarizable Harish-Chandra module of $\tilde{O}(n, 1)$. This will imply that $t_{\lambda, \lambda^{\prime}}$ and $T_{\lambda, \lambda^{\prime}}$ are never zero. This will in turn prove that $\Omega(1)$ is irreducible. These will be done in $\$ 5$. Unfortunately, it involves a case-by-case consideration.

We remark that hidden in the background of these proofs is the use of GelfandZetlin bases of irreducible representations of $\mathrm{SO}(n)$ GZ. Indeed using these bases, we can construct bases of $\Omega(1)$ and $\Omega(1, \mu)$ with explicit Lie algebra actions.

1.7. This paper is partly motivated by [LZ1]. In [Zhu and LZ1, they investigate the Howe quotient lifts of one-dimensional unitary characters of $\widetilde{\mathrm{U}}(p, q)$ to $\widetilde{\mathrm{U}}(n, n)$ and they show that the Howe quotients can be embedded into a degenerate principal series representation of $\widetilde{\mathrm{U}}(n, n)$. Several authors have used transition coefficients to investigate $K$-multiplicity free principal series representations. See [Hi], HT, [KG], [J], Le], Sa, [Zha].

1.8. There were many investigations on the theta lifts and the Howe quotients which are lifts from one-dimensional unitary representations and discrete series representations, especially when the dual pair is in the stable range. See 2.5 for the definition of the stable range. Some results in this area are $\underline{\mathrm{Ho}}$, , $\mathrm{KoO}$, [KR, [Li1, [Li2, [LZ1, [LZ2], [NZ1, [TZ, [Tan, [Zhu, [ZH] and many more. We will need some of these results and we will give a fuller account of them as we go along.

1.9. The organization of this paper is as follows. Section 2 introduces some notation about Howe correspondences. Section 3 calculates the $K_{n, m}$-types of $\Omega_{p}^{n, m}(1)$. Section 4 proves (1). In $\$ 5$ we introduce the transition coefficients. The proofs of Theorems 1.2.1 1.5.1 and 1.5.2 will occupy $\$ 6$ and 88 In 99 we identify some of the unitarizable theta lifts with Vogan-Zuckerman's $A_{\mathfrak{q}}(\lambda)$. Finally, in Appendix A, Soo Teck Lee computes the composition series of the Howe quotients lifted from the unitary one-dimensional genuine representations for the dual pairs $(\widetilde{\mathrm{Sp}}(p, \mathbb{R}), \tilde{\mathrm{O}}(n, n))$.

1.10. Dual pairs $(\widetilde{\mathrm{U}}(p, q), \widetilde{\mathrm{U}}(n, m))$ and $\left(\tilde{\mathrm{O}}^{*}(2 p), \widetilde{\mathrm{Sp}}(n, m)\right)$. We have mentioned in the beginning that the method employed in this paper also works for the Howe quotients of one-dimensional genuine unitary characters and genuine unitary lowest weight modules appearing for the dual pair $(\widetilde{\mathrm{U}}(p, q), \widetilde{\mathrm{U}}(n, m))$. The same works for the dual pair $\left(\tilde{\mathrm{O}}^{*}(2 p), \widetilde{\mathrm{Sp}}(n, m)\right)$ except for one family of unitary lowest weight modules of $\tilde{\mathrm{O}}^{*}(2 p)$ which does not occur in the compact dual pair correspondences DER.

Results on the three dual pairs were presented in a first draft of this paper. Indeed all the results and proofs for the dual pair $(\widetilde{\mathrm{Sp}}(p, \mathbb{R}), \tilde{\mathrm{O}}(n, m))$ in this paper for (11), Theorems 1.5.1 and 1.5.2 also hold for the other two dual pairs. Unfortunately, there are enough differences so that separate notations and proofs are rendered for most parts. We prefer to leave these to the reader. 
For the dual pair $\left(\tilde{\mathrm{O}}^{*}(2 p), \widetilde{\mathrm{Sp}}(n, m)\right)$, the computation of the submodules of the Howe quotients follow a similar idea as that of the dual pair $(\widetilde{\mathrm{Sp}}(p, \mathbb{R}), \tilde{\mathrm{O}}(n, m))$. There are less cases to consider and the results are less complicated.

For the dual pair $(\widetilde{\mathrm{U}}(p, q), \widetilde{\mathrm{U}}(n, m))$ the Howe quotient is reducible outside the stable range. In order to determine its composition series, we need a detailed analysis of the transition coefficients and the proofs quickly degenerate into long and tedious case-by-case computations. Therefore we will focus our attention on the dual pair $\widetilde{\mathrm{Sp}}(p, \mathbb{R}) \times \widetilde{\mathrm{O}}(n, m)$ in this paper and we postpone the dual pair $(\widetilde{\mathrm{U}}(p, q), \widetilde{\mathrm{U}}(n, m))$ to a future paper.

While we were writing this paper, we received the preprint [PT] which computes the theta lifts of unitary characters with respect to the dual pair $(\widetilde{\mathrm{U}}(p, q), \widetilde{\mathrm{U}}(n, m))$.

Acknowledgments. The author would like to thank Soo Teck Lee whose valuable discussions and generous comments have made this paper possible, and for allowing the inclusion of his results on $\tilde{\mathrm{O}}(n, n)$ in the appendix. We would like to thank the first referee, K. Nishiyama, D. Prasad, T. Przebinda, and C-B. Zhu for their valuable comments on the first draft.

\section{Howe QUOTIENTS LifTed FROM ONE-Dimensional UNITARY REPRESENTATIONS}

2.1. The Fock Model. Let $\widetilde{\operatorname{Sp}}(N, \mathbb{R})$ denote the double cover of $\operatorname{Sp}(N, \mathbb{R})$. Let $\omega_{N}$ denote the Fock model of the Weil representation of $\widetilde{\operatorname{Sp}}(N, \mathbb{R})$. It is a well-known fact that

$$
\omega_{N+M}=\omega_{N} \otimes \omega_{M}
$$

as an infinitesimal representation of $\widetilde{\operatorname{Sp}}(N, \mathbb{R}) \times \widetilde{\operatorname{Sp}}(M, \mathbb{R})$.

Suppose $G_{i}(i=1,2)$ is a two-fold cover of an algebraic group $\bar{G}_{i}$. Let $C_{i} \simeq \mathbb{Z} / 2 \mathbb{Z}$ denote the kernel of the covering map. Then we define

$$
G_{1} \times_{2} G_{2}:=\left(G_{1} \times G_{2}\right) /\left\{(x, x) \in C_{1} \times C_{2}: x \in \mathbb{Z} / 2 \mathbb{Z}\right\} .
$$

2.2. A see-saw pair. We consider the following dual pairs:

$$
\begin{aligned}
& \widetilde{\mathrm{Sp}}(p, \mathbb{R})^{\prime} \times{ }_{2} \tilde{\mathrm{O}}(n, r) \subset \widetilde{\mathrm{Sp}}(p(n+r), \mathbb{R}), \\
& \widetilde{\mathrm{Sp}}(p, \mathbb{R})^{\prime \prime} \times{ }_{2} \tilde{\mathrm{O}}(m-r) \subset \widetilde{\mathrm{Sp}}(p(m-r), \mathbb{R}), \\
& \widetilde{\mathrm{Sp}}(p, \mathbb{R}) \times{ }_{2} \tilde{\mathrm{O}}(n, m) \subset \widetilde{\mathrm{Sp}}(p(n+m), \mathbb{R}) .
\end{aligned}
$$

Note that $\widetilde{\operatorname{Sp}}(p, \mathbb{R})$ splits over $\operatorname{Sp}(p, \mathbb{R})$ if and only if $m+n$ is even. Likewise, for $\widetilde{\mathrm{Sp}}(p, \mathbb{R})^{\prime}$ and $\widetilde{\mathrm{Sp}}(p, \mathbb{R})^{\prime \prime}$. The Lie group $\tilde{\mathrm{O}}(n, m)$ is the double cover of $\mathrm{O}(n, m)$ defined by

$$
\tilde{\mathrm{O}}(n, m):=\left\{(g, z) \in \mathrm{O}(n, m) \times \mathbb{C}^{*}: \operatorname{det}^{p}(g)=z^{2}\right\} .
$$

Let $\xi_{n, m}$ denote the character of $\tilde{O}(n, m)$ given by $\xi_{n, m}((g, z))=z$. Similarly, we define $\tilde{\mathrm{O}}(r)$ and its character $\xi_{r}$ by setting $m=0$ and $n=r$ in the above formulas.

The dual pairs form a see-saw pair

$$
\begin{array}{rll}
\widetilde{\operatorname{Sp}}(p, \mathbb{R})^{\prime} \times{ }_{2} \widetilde{\operatorname{Sp}}(p, \mathbb{R})^{\prime \prime} & & \tilde{\mathrm{O}}(n, m) \\
\widetilde{\operatorname{Sp}}(p, \mathbb{R}) & & \tilde{\mathrm{O}}(n, r) \times_{2} \tilde{\mathrm{O}}(m-r) .
\end{array}
$$


2.3. Howe quotients. We will recall some definitions and facts from [Ho2]. Let $G_{1} \times_{2} G_{2} \subset \widetilde{\operatorname{Sp}}(N, \mathbb{R})$ denote one of the three dual pairs in (2) to (44). Let $\mathfrak{g}_{(i)}$ and $K_{(i)}$ denote the Lie algebra and maximal compact subgroup of $G_{i}(i=1,2)$ respectively. Note that $G_{(i)}$ is a double cover of a real algebraic group $\bar{G}_{(i)}$. Let $\pi_{1}$ denote an irreducible $\left(\mathfrak{g}_{(1)}, K_{(1)}\right)$-module. We call $\pi_{1}$ a genuine $\left(\mathfrak{g}_{(1)}, K_{(1)}\right)$-module if the kernel of the covering map $G_{(1)} \rightarrow \bar{G}_{(1)}$ acts nontrivially.

We define

$$
\mathcal{N}=\bigcap\left\{\operatorname{ker} f: f \in \operatorname{Hom}_{\left(\mathfrak{g}_{(1)}, K_{(1)}\right)}\left(\omega_{N}, \pi_{1}\right)\right\} .
$$

Then

$$
\omega_{N} / \mathcal{N}=\Omega\left(\pi_{1}\right) \otimes \pi_{1}
$$

for some admissible $\left(\mathfrak{g}_{(2)}, K_{(2)}\right)$-module $\Omega\left(\pi_{1}\right)$ of finite length. We will call $\Omega\left(\pi_{1}\right)$ the (maximal) Howe quotient lifted from $\pi_{1}$ with respect to the dual pair $G_{1} \times G_{2}$. It is nonzero only if $\pi_{1}$ is a genuine $\left(\mathfrak{g}_{(1)}, K_{(1)}\right)$-module. By Lemma 2.2 of [Ho2, $\Omega\left(\pi_{1}\right)$ is the genuine $\left(\mathfrak{g}_{(2)}, K_{(2)}\right)$-module of the Howe quotient defined using the smooth model of the Weil representation. By a theorem of Howe, $\Omega\left(\pi_{1}\right)$ has a unique irreducible quotient $\theta\left(\pi_{1}\right)$ called the (local) theta lift and $\theta\left(\pi_{1}\right)$ is uniquely determined by $\pi_{1}$.

We claim that

$$
\Omega\left(\pi_{1}\right)^{*}=\operatorname{Hom}_{\left(\mathfrak{g}_{(1)}, K_{(1)}\right)}\left(\omega_{N}, \pi_{1}\right)_{K_{(2)}} .
$$

Indeed

$$
\begin{aligned}
& \operatorname{Hom}_{\left(\mathfrak{g}_{(1)}, K_{(1)}\right)}\left(\left(\omega_{N}\right), \pi_{1}\right)_{K_{(2)}}=\operatorname{Hom}_{\left(\mathfrak{g}_{(1)}, K_{(1)}\right)}\left(\left(\omega_{N}\right) / \mathcal{N}, \pi_{1}\right)_{K_{(2)}} \\
& \quad=\operatorname{Hom}_{\left(\mathfrak{g}_{(1)}, K_{(1)}\right)}\left(\Omega\left(\pi_{1}\right) \otimes \pi_{1}, \pi_{1}\right)_{K_{(2)}}=\operatorname{Hom}_{\mathbb{C}}\left(\Omega\left(\pi_{1}\right), \mathbb{C}\right)_{K_{(2)}}=\Omega\left(\pi_{1}\right)^{*} .
\end{aligned}
$$

2.4. We note that $\widetilde{\operatorname{Sp}}(p, \mathbb{R})$ will exhibit a genuine one-dimensional character $\xi_{\mathrm{s}}$ if and only if it is a split cover over $\operatorname{Sp}(p, \mathbb{R})$, that is,

$$
\widetilde{\mathrm{Sp}}(p, \mathbb{R})=\operatorname{Sp}(p, \mathbb{R}) \times(\mathbb{Z} / 2 \mathbb{Z}) .
$$

This happens precisely when $n+m$ is even and $\xi_{\mathrm{s}}$ is the character of $\widetilde{\operatorname{Sp}}(p, \mathbb{R})$ which is nontrivial on $\mathbb{Z} / 2 \mathbb{Z}$ and trivial on $\operatorname{Sp}(p, \mathbb{R})$.

We will abuse notation and we denote the Howe quotient $\Omega\left(\xi_{\mathrm{s}}\right)$ lifted from $\widetilde{\mathrm{Sp}}(p, \mathbb{R})$ to $\tilde{\mathrm{O}}(n, m)$ by $\Omega(1)$ or $\Omega_{p}^{n, m}(1)$.

We remark that since $m+n$ is even, $\widetilde{\mathrm{Sp}}(p, \mathbb{R})^{\prime}$ and $\widetilde{\mathrm{Sp}}(p, \mathbb{R})^{\prime \prime}$ in (2) and (3) are either both split or both nonsplit. Hence $\widetilde{\operatorname{Sp}}(p, \mathbb{R})^{\prime}$ and $\widetilde{\operatorname{Sp}}(p, \mathbb{R})^{\prime \prime}$ are isomorphic Lie groups.

2.5. We recall that the dual pair $(\widetilde{\mathrm{Sp}}(p, \mathbb{R}), \tilde{\mathrm{O}}(n, m))$ is said to be in the stable range if $2 p \leq \min (n, m)$. We recall Theorem 2.2 in $[\mathrm{ZH}]$ in which Case (I) in Theorem 1.2 .1 is a special case.

Theorem 2.5.1. Suppose $\left(G_{1}, G_{2}\right)$ is a reductive dual pair in the stable range with $G_{1}$ being the smaller group and suppose it is not the dual pair $(\widetilde{\mathrm{Sp}}(p, \mathbb{R}), \tilde{\mathrm{O}}(2 p, 2 p))$. Then the Howe quotient $\Omega(1)$ lifted from the order 2 genuine character of $G_{1}$ is a nonzero irreducible and unitarizable Harish-Chandra module of $\tilde{\mathrm{O}}(n, m)$.

We remark that Zhu and Huang prove that $\Omega(1)$ is irreducible for all dual pairs in the stable range. It is nonzero and unitarizable, as follows from a theorem of $\mathrm{Li}$ Li1. 


\section{3. $K$-TYPES OF $\Omega(1)$}

In this section, we recall the construction of unitary lowest weight modules via dual pair correspondences due to Kashiwara-Vergne [KV]. Next we will use this to calculate the $K_{n, m}$-types of $\Omega(1)$. First we need to set up some notations.

\subsection{Notations. Define}

$$
\Lambda(n):=\left\{\lambda=\left(\lambda_{1}, \ldots, \lambda_{n}\right) \in \mathbb{Z}^{n}: \lambda_{i} \geq \lambda_{i+1}\right\} .
$$

Let $\Lambda^{+}(n)$ be the subset consisting of those $\lambda$ 's such that $\lambda_{i} \geq 0$. Sometimes we will write $\left(\lambda_{1}, \ldots, \lambda_{n}\right)_{n}$ with a subscript $n$ outside the parenthesis to indicate that it belongs to $\mathbb{Z}^{n}$. We define $\varepsilon_{i}:=(0, \ldots, 0,1,0, \ldots, 0)$ where 1 appears in the $i$-th position, $\mathbf{1}_{n}:=(1,1, \ldots, 1)_{n}, \mathbf{0}_{n}:=(0,0, \ldots, 0)_{n}$ and $\frac{1}{2} \Lambda(n):=\left\{\frac{1}{2} \lambda: \lambda \in \Lambda(n)\right\}$. If $\lambda=\left(\lambda_{1}, \ldots, \lambda_{n}\right) \in \mathbb{Z}^{n}$ and $\lambda^{\prime}=\left(\lambda_{1}^{\prime}, \ldots, \lambda_{m}^{\prime}\right) \in \mathbb{Z}^{n}$, we abuse notation and denote $\left(\lambda_{1}, \ldots, \lambda_{n}, \lambda_{1}^{\prime}, \ldots, \lambda_{m}^{\prime}\right) \in \mathbb{Z}^{n+m}$ by $\left(\lambda, \lambda^{\prime}\right)$.

3.2. Highest weights. Irreducible representations of $\mathrm{O}(n)$ are parameterized by elements $\lambda$ in $\Lambda^{+}(n)$ of the form

$$
\begin{aligned}
\lambda & =\left(a_{1}, \ldots, a_{k}, 0, \ldots, 0\right)_{n} \text { or } \\
\lambda & =\left(a_{1}, \ldots, a_{k}, 1, \ldots, 1,0, \ldots, 0\right)_{n}
\end{aligned}
$$

where $a_{i}$ are positive integers, and $k \leq\left[\frac{n}{2}\right]$ (see [Ad], [GoW]). In (8) there are $n-2 k$ copies of "1". Let $\bar{\tau}_{n}^{\lambda}$ denote the finite-dimensional representation corresponding to $\lambda$. Then $\xi_{n} \bar{\tau}_{n}^{\lambda}$ is a genuine representation of $\tilde{\mathrm{O}}(n)$ and we denote it by $\tau_{n}^{\lambda}$. We call (7) and (8) genuine highest weights of $\tilde{O}(n)$. Sometimes we will abuse notation and write $\lambda$ in place of $\tau_{n}^{\lambda}$. We also let $(\tilde{\tilde{O}(n)})_{\text {gen }}$ denote both the set of irreducible genuine representations of $\tilde{O}(n)$ and the corresponding set of genuine highest weights. Note that

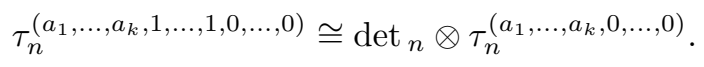

The group $\tilde{\mathrm{O}}(n)$ contains $\mathrm{SO}(n)$ and $\xi_{n}$ is trivial on $\mathrm{SO}(n)$. The restriction of $\tau_{n}^{\lambda}$ (and also $\bar{\tau}_{n}^{\lambda}$ ) to $\mathrm{SO}(n)$ is reducible iff $n=2 k$ is even. In this case the restriction decomposes into two irreducible representations of $\mathrm{SO}(n)$ of weights

$$
\left(a_{1}, \ldots, a_{k}\right)_{k} \text { and }\left(a_{1}, \ldots, a_{k-1},-a_{k}\right)_{k} .
$$

Otherwise, $\tau_{n}^{\lambda}$ is the irreducible representation of $\mathrm{SO}(n)$ with highest weight

$$
\left(a_{1}, \ldots, a_{k}, 0, \ldots, 0\right)_{[n / 2]} .
$$

3.3. Let $\widetilde{\mathrm{U}}(p)^{\prime \prime}$ denote the maximal compact subgroup of $\widetilde{\operatorname{Sp}}(p, \mathbb{R})^{\prime \prime}$ in (3). Then $\widetilde{\mathrm{U}}(p)^{\prime \prime}$ exhibits a nontrivial genuine one-dimensional character $\xi_{p}^{\prime \prime}$ such that $\left(\xi_{p}^{\prime \prime}\right)^{2}=$ $\operatorname{det}_{p}^{m-r}$. Suppose $W$ is an irreducible genuine representation of $\mathrm{U}(p)^{\prime \prime}$. Then the map $W \mapsto \xi_{p}^{\prime \prime} W$ defines a bijection between the set of irreducible representations of $\mathrm{U}(p)$ and the set of genuine irreducible representations of $\widetilde{\mathrm{U}}(p)^{\prime \prime}$. Therefore we define $\Lambda(p)+\frac{m-r}{2} \mathbf{1}_{p}$ to be the set of highest weights of genuine representations of $\widetilde{\mathrm{U}}(p)^{\prime \prime}$. 
3.4. Unitary lowest weight modules. Let $\tau_{m-r}^{\mu}$ be an irreducible genuine representation of $\tilde{\mathrm{O}}(m-r)$ with highest weight $\mu$. Let $\left(\omega_{M}\right)_{\mu}$ denote the $\tau_{m-r}^{\mu}$ isotypic component of $\omega_{N}$ with respect to the dual pair (3). It is well known that if it is nonzero, then (see $[\mathrm{KV}]$ )

$$
\left(\omega_{M}\right)_{\mu}=\Theta(\mu) \otimes \tau_{m-r}^{\mu}
$$

where $\Theta(\mu)$ is an irreducible lowest weight module of $\widetilde{\operatorname{Sp}}(p, \mathbb{R})^{\prime \prime}$. Suppose $\mu=$ $\left(a_{1}, \ldots, a_{k}, 0, \ldots, 0\right)_{n} \in \Lambda^{+}(n)$, then the highest weight of the lowest $\widetilde{\mathrm{U}}(p)^{\prime \prime}$-type of $\Theta(\mu)$ is (See $[\mathrm{KV}]$ and $[\mathrm{Ad}]$ ).

$$
\left(a_{1}, \ldots, a_{k}, 0, \ldots, 0\right)_{p}+\frac{n}{2} \mathbf{1}_{p} .
$$

3.5. We will compute the $K_{n, m}$-types of $\Omega(1)$. Let $\tau_{n}^{\lambda} \otimes \tau_{m}^{\kappa}$ denote an irreducible genuine representation of $K_{n, m}=\tilde{\mathrm{O}}(n) \times_{2} \tilde{\mathrm{O}}(m)$. Let $\Theta(\lambda)$ (resp. $\left.\Theta(\kappa)\right)$ denote the theta lift with respect to the dual pair $\left(\tilde{\mathrm{O}}(n), \widetilde{\mathrm{Sp}}(p, \mathbb{R})^{\prime}\right)\left(\operatorname{resp} .\left(\tilde{\mathrm{O}}(m), \widetilde{\mathrm{Sp}}(p, \mathbb{R})^{\prime \prime}\right)\right)$. Let $\gamma$ (resp. $\left.\gamma^{\prime}\right)$ denote the lowest $\widetilde{\mathrm{U}}(p)^{\prime}$-type of $\Theta(\lambda)$ (resp. lowest $\widetilde{\mathrm{U}}(p)^{\prime \prime}$-type of $\Theta(\kappa))$.

Lemma 3.5.1. The multiplicity of $\tau_{n}^{\lambda} \otimes \tau_{m}^{\kappa}$ in $\Omega(1)$ is either one or zero. It is one iff $\gamma=\gamma^{\prime}$ as representations of $\widetilde{\mathrm{U}}(p)^{\prime}$.

Proof. Also see [KR], [LZ1 and [NZ1. We set $r=0$ in the see-saw pair in (5) and the usual see-saw pair argument shows that

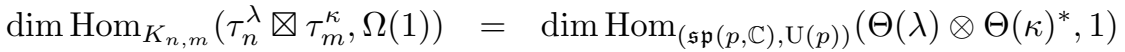

$$
\begin{aligned}
& =\operatorname{dim} \operatorname{Hom}_{\left(\mathfrak{s p}(p, \mathbb{C}), \widetilde{\mathrm{U}}(p)^{\prime}\right)}(\Theta(\lambda), \Theta(\kappa)) \\
& =\operatorname{dim} \operatorname{Hom}_{\widetilde{\mathrm{U}}(p)^{\prime}}\left(\gamma, \gamma^{\prime}\right) \text {. }
\end{aligned}
$$

Applying the Schur's lemma to the last equation proves the lemma.

Combining the above lemma with (11) gives the next lemma.

Lemma 3.5.2. The Howe quotient $\Omega_{p}^{n, m}(1)$ is $K_{n, m}$-multiplicity free and it is the sum of all $K_{n, m}$-types of the form

$$
\left(a_{1}, \ldots, a_{k}, 0, \ldots, 0\right)_{n} \otimes\left(a_{1}^{\prime}, \ldots, a_{k^{\prime}}^{\prime}, 0, \ldots, 0\right)_{m}
$$

where $a_{i}, a_{i}^{\prime}$ are positive integers, $a_{i} \geq a_{i+1}$ and $a_{i}^{\prime} \geq a_{i+1}^{\prime}$ and

$$
\left(a_{1}^{\prime}, \ldots, a_{k^{\prime}}^{\prime}, 0, \ldots, 0\right)_{p}=\left(a_{1}, \ldots, a_{k}, 0, \ldots, 0\right)_{p}+\frac{n-m}{2} \mathbf{1}_{p} .
$$

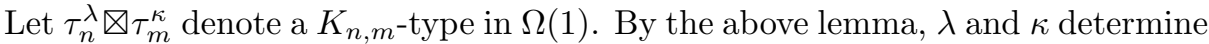
each other linearly and uniquely. Sometimes we write $\kappa$ as $\kappa(\lambda)$ to indicate that it is a linear function of $\lambda$. We will denote the $K_{n, m}$-type $\tau_{n}^{\lambda} \otimes \tau_{m}^{\kappa}$ by $\tau_{n, m}^{\lambda}$ and we write

$$
\Omega(1)=\sum_{\lambda \in J} \tau_{n, m}^{\lambda}=\sum_{\lambda \in J} \tau_{n}^{\lambda} \otimes \tau_{m}^{\kappa(\lambda)}
$$

where $J$ is a subset of $(\widehat{\widetilde{O}(n)})_{\text {gen }}$. 
Suppose $\Omega(1)$ is in Cases (I), (II) or (III) in Theorem 1.2.1 and we denote the index $J$ in (14) above as $J_{I}, J_{I I}$ and $J_{I I I}$ respectively. It is easy to see that

$$
\begin{aligned}
J_{I} & =\left\{\left(a_{1}, \ldots, a_{p}, 0, \ldots, 0\right) \in \Lambda^{+}(n): a_{p} \geq \frac{m-n}{2}\right\}, \\
J_{I I} & =\left\{\left(a_{1}, \ldots, a_{\min (n, p)}, 0, \ldots, 0\right) \in(\widehat{\mathrm{O}(n)})_{\mathrm{gen}}: a_{i} \geq 0\right\}, \\
J_{I I I} & =\left\{\left(a_{1}, \ldots, a_{p}, 0, \ldots, 0\right) \in \Lambda^{+}(n): a_{n-p+1}=\ldots=a_{p}=1\right\} .
\end{aligned}
$$

This proves the claim about $\Omega(1) \neq 0$ in these three cases.

We claim that $\Omega_{p}^{n, m}(1)=0$ outside the above three cases. Indeed, suppose $\Omega(1) \neq 0$ and we are not in Cases (I) and (II), then by (13) $a_{k}>0$ and $p=k \leq$ $n<m$ and $n<2 p$. Since $\left[\frac{n}{2}\right]<p, a_{p}=1$. This shows that $\frac{m-n}{2}=1$ and $m=n+2$, that is, Case (III).

The following is an important corollary of (14).

Corollary 3.5.3. The Howe quotient $\Omega_{p}^{n, m}(1)$ lifted from $\widetilde{\mathrm{Sp}}(p, \mathbb{R})$ to $\tilde{\mathrm{O}}(n, m)$ is $\tilde{\mathrm{O}}(n) \times 1$-admissible.

\section{Howe Quotient Lifted from $\Theta(\mu)$}

4.1. Recall $\$ 2.2$ that $\tilde{\mathrm{O}}(n, m)$ contains $\tilde{\mathrm{O}}(n, r) \cdot \tilde{\mathrm{O}}(m-r)$ and $K_{n, r} \cdot \tilde{\mathrm{O}}(m-r) \subset$ $K_{n, m}$. In this section, we do not assume that $n \leq m$.

Let $\Omega(1)_{\mu}$ denote the $\tau_{m-r}^{\mu}$ isotypic component of $\Omega(1)$. By Corollary 3.5.3 this is an (admissible) Harish-Chandra module of $\tilde{\mathrm{O}}(n, r) \times \tilde{\mathrm{O}}(m-r)$. By Proposition 1.6 [Ko2, the subspaces of $K_{n, r}$-finite vectors and $K_{n, m}$-finite vectors agree and (6) becomes

$$
\Omega(1)^{*}=\operatorname{Hom}_{(\mathfrak{s p}(p, \mathbb{C}), \widetilde{\mathrm{U}}(p))}\left(\omega_{(p(n+m))}, \xi_{\mathrm{s}}\right)_{K_{n, r}} .
$$

4.2. We recall in $₫ 2.4$ that $\widetilde{\mathrm{Sp}}(p, \mathbb{R})^{\prime}$ and $\widetilde{\mathrm{Sp}}(p, \mathbb{R})^{\prime \prime}$ are isomorphic. Let $\Theta(\mu)$ be the irreducible genuine lowest weight module of $\widetilde{\mathrm{Sp}}(p, \mathbb{R})^{\prime \prime}$ lifted from $\tau_{m-r}^{\mu}$ of $\tilde{\mathrm{O}}(m-r)$ in (10). We define $\Omega(1, \mu)$ to be the Howe quotient lifted from $\Theta(\mu)$ with respect to the dual pair $\left(\widetilde{\mathrm{Sp}}(p, \mathbb{R})^{\prime}, \tilde{\mathrm{O}}(n, r)\right)$.

We will prove (11) which is the key lemma in this paper.

Lemma 4.2.1. Let $\mu$ denote the highest weight of the representation $\tau_{m-r}^{\mu}$ and we define $\Omega(1, \mu)$ as above. Then (also see (1))

$$
\Omega_{p}^{n, m}(1)_{\mu}=\Omega_{p}^{n, r}(1, \mu) \otimes \tau_{m-r}^{\mu}
$$

as a Harish-Chandra module of $\tilde{\mathrm{O}}(n, r) \times \tilde{\mathrm{O}}(m-r)$.

Proof. Note that $\tau_{m-r}^{\mu}$ is a self-dual representation. Let $\widetilde{\mathrm{U}}(p)$ be the maximal compact subgroup of $\widetilde{\operatorname{Sp}}(p, \mathbb{R})$. Set $N=p(n+r)$ and $M=p(m-r)$. By (18)

$$
\begin{aligned}
\left(\Omega(1)_{\mu}\right)^{*} & =\left(\Omega(1)^{*}\right)_{\mu}=\operatorname{Hom}_{(\mathfrak{s p}(p, \mathbb{C}), \widetilde{\mathrm{U}}(p))}\left(\left(\omega_{N+M}\right)_{\mu}, \xi_{\mathrm{s}}\right)_{K_{n, r}} \\
& =\operatorname{Hom}_{(\mathfrak{s p}(p, \mathbb{C}), \mathrm{U}(p))}\left(\omega_{N} \otimes\left(\omega_{M}^{*}\right)_{\mu}, 1\right)_{K_{n, r}} .
\end{aligned}
$$

The character $\xi_{\mathrm{s}}$ is replaced by the trivial character in the second equation because the action of $\widetilde{\mathrm{U}}(p)$ on $\omega_{N} \otimes\left(\omega_{M}^{*}\right)$ factors through $\mathrm{U}(p)$.

$$
\begin{aligned}
\text { Eqn (19) } & \left.=\operatorname{Hom}_{(\mathfrak{s p}(p, \mathbb{C}), \mathrm{U}(p))}\left(\omega_{N} \otimes(\Theta(\mu))^{*} \otimes \tau_{m-r}^{\mu}, 1\right)_{K_{n, r}} \quad \text { (By (10) }\right) \\
& =\operatorname{Hom}_{\left(\mathfrak{s p}(p, \mathbb{C}), \widetilde{\mathrm{U}}(p)^{\prime}\right)}\left(\omega_{N}, \Theta(\mu)\right)_{K_{n, r}} \otimes \tau_{m-r}^{\mu} .
\end{aligned}
$$

Note that the last equality holds because $\Theta(\mu)$ is $\widetilde{\mathrm{U}}(p)$-admissible. 
Corollary 4.2.2. Let $\Omega(1), \tau_{m-r}^{\mu}$ and $\Theta(\mu)$ as in $\$ 3.4$ and 4.1 . Then the local theta lift $\theta(\Theta(\mu))$ of the unitary lowest weight module $\Theta(\mu)$ of $\widetilde{\mathrm{Sp}}(p, \mathbb{R})$ to $\tilde{\mathrm{O}}(n, r)$ is nonzero if and only if:

(i) $\Omega(1)$ is nonzero, and

(ii) $\Omega(1)$ contains a $K_{n, m}$-type $\tau_{n, m}^{\lambda}$ (see (14)) whose restriction to $1 \times \tilde{\mathrm{O}}(m-r)$ contains $\tau_{m-r}^{\mu}$ as a subrepresentation.

The corollary is evident from the last lemma. It is relatively easy to check conditions (i) and (ii) in the above corollary using the branching rules in Lemma 5.1 .2 in the next section.

We remark that in the case when $\Theta(\mu)$ is a discrete series representation with sufficiently regular infinitesimal character and $2 p \leq n+r$, then Li Li2 showed that $\Omega(1, \mu)$ is irreducible and unitarizable and it has nonzero $\left(\mathfrak{s o}(n, r), K_{n, r}\right)$ cohomology $\mathrm{VZ}$. Also see some related recent articles of $\mathrm{He} \mathrm{He} 1, \mathrm{He} 2$.

4.3. The next corollary is a special case of Theorems 1.5.1 and 1.5.2, However, we can give a simple and direct proof. Later we will use this to prove Case (III) in Theorem 1.2.1.

Corollary 4.3.1. If $\Omega(1)$ is a unitarizable (and hence irreducible) Harish-Chandra module of $\tilde{\mathrm{O}}(n, m)$ and $\Omega(1, \mu)$ is nonzero, then $\Omega(1, \mu)$ is an irreducible and unitarizable $\left(\mathfrak{s o}(n, r), K_{n, r}\right)$-module. In particular, $\Omega(1, \mu)$ is the local theta lift.

Proof. The Howe quotient $\Omega(1, \mu)$ inherits the Hermitian form from $\Omega(1)$ and it is therefore unitarizable. On the other hand, $\Omega(1, \mu)$ is a cyclic $\left(\mathfrak{s o}(n, r), K_{n, r}\right)$-module of finite length with a unique quotient [Ho2 . Hence it is completely decomposable. This implies that it is irreducible. The same argument also shows that $\Omega(1)$ is irreducible.

\section{Transition Coefficients}

The study of the reducibility and unitarizability of $\Omega(1)$ is closely related to the computations of the transition coefficients which we will define in this section.

5.1. Branching rules and tensor products. We will state some well-known results on the finite-dimensional representations of $\mathrm{O}(n)$. Let $\bar{\tau}_{n}^{\lambda}$ denote an irreducible representation of $\mathrm{O}(n)$ with highest weight $\lambda=\left(\lambda_{1}, \ldots, \lambda_{n}\right)_{n} \in \Lambda^{+}(n)$. The corresponding statements about its double cover $\tilde{\mathrm{O}}(n)$ are obtained by tensoring $\bar{\tau}_{n}^{\lambda}$ with the genuine one-dimensional character $\xi_{n}$.

Lemma 5.1.1. Let $\bar{\tau}_{n}^{\lambda}$ and $\bar{\tau}_{n-1}^{\lambda^{\prime}}$ denote irreducible representations of $\mathrm{O}(n)$ and $\mathrm{O}(n-1)$ respectively. Let $\chi_{1}$ denote the nontrivial order 2 character of $\mathrm{O}(1)=$ $\mathbb{Z} / 2 \mathbb{Z}$. Then the restriction of $\bar{\tau}_{n}^{\lambda}$ to $\mathrm{O}(n-1) \times \mathrm{O}(1)$ contains the irreducible representation $\bar{\tau}_{n-1}^{\left(\lambda_{1}^{\prime}, \ldots, \lambda_{n-1}^{\prime}\right)} \otimes \chi_{1}^{s}$ with multiplicity at most one. It is one iff

(i) $\lambda_{1} \geq \lambda_{1}^{\prime} \geq \lambda_{2} \geq \ldots \geq \lambda_{n-1}^{\prime} \geq \lambda_{n}$, and

(ii) $s=\sum_{i=1}^{n} \lambda_{i}-\sum_{i=1}^{n-1} \lambda_{i}^{\prime}$.

The above lemma is well known. For a proof, see $\S 10, \underline{\mathrm{Pr}}$ and $\S 5.4 .2 .3, \underline{\mathrm{Ho} 3}$. The next lemma is a simple consequence. 
Lemma 5.1.2. Let $0<r<n$ and let $\bar{\tau}_{n}^{\lambda}$ and $\bar{\tau}_{n-r}^{\lambda^{\prime}}$ denote irreducible representation of $\mathrm{O}(n)$ and $\mathrm{O}(n-r)$ respectively. Then $\bar{\tau}_{n}^{\lambda}$ contains $\bar{\tau}_{n-r}^{\lambda^{\prime}}$ iff $\lambda_{i} \geq \lambda_{i}^{\prime} \geq \lambda_{i+r}$. Here we set $\lambda_{i}=-\infty$ if $i>n$.

Lemma 5.1.3. Let $\mathbb{C}^{n}$ denote the standard representation of $\mathrm{O}(n)$. Then

$$
\mathbb{C}^{n} \otimes \bar{\tau}_{n}^{\lambda}=\sum_{i=1}^{n} \bar{\tau}_{n}^{\lambda+\varepsilon_{i}}+\sum_{i=1}^{n} \bar{\tau}_{n}^{\lambda-\varepsilon_{i}} .
$$

Here we assume that $\bar{\tau}_{n}^{\lambda \pm \varepsilon_{i}}=0$ if $\lambda \pm \varepsilon_{i}$ is not a highest weight of $\mathrm{O}(n)$.

Proof. The corresponding results are well known for $\mathrm{SO}(n)$. One can deduce the results for $\mathrm{O}(n)$ from that of $\mathrm{SO}(n)$ and we will leave this as an exercise for the reader.

The following corollary follows by applying Lemma 5.1.1 to Lemma 4.2.1.

\section{Corollary 5.1.4.}

$$
\operatorname{Res}_{\left(\mathfrak{s o}(n, r-1), K_{n, r-1}\right)}^{\left(\mathfrak{s o}(n, r), K_{n, r}\right)} \Omega(1, \mu)=\sum_{\mu^{\prime}} \Omega(1, \mu)
$$

where the sum is taken over all highest weights $\mu^{\prime}=\left(\mu_{1}^{\prime}, \ldots, \mu_{r+1}^{\prime}\right)$ of $\tilde{\mathrm{O}}(m-r+1)$ such that

$$
\mu_{1}^{\prime} \geq \mu_{1} \geq \mu_{2}^{\prime} \geq \mu_{2} \geq \ldots \geq \mu_{m-r} \geq \mu_{m-r+1}^{\prime} \geq 0 .
$$

5.2. Transition coefficients. Let $\mathfrak{s o}(n, m)_{\mathbb{C}}=\left(\mathfrak{k}_{n, m}\right)_{\mathbb{C}} \oplus \mathfrak{p}_{1}$ denote the Cartan decomposition of the complexified Lie algebra of $\tilde{\mathrm{O}}(n, m)$. As a representation of $\bar{K}_{n, m}=\mathrm{O}(n) \times \mathrm{O}(m), \mathfrak{p}_{1}=\mathbb{C}^{n} \otimes \mathbb{C}^{m}$.

Next suppose $\tau_{n, m}^{\lambda}=\tau_{n}^{\lambda} \otimes \tau_{m}^{\kappa(\lambda)}$ and $\tau_{n, m}^{\lambda^{\prime}}=\tau_{n}^{\lambda^{\prime}} \otimes \tau_{m}^{\kappa\left(\lambda^{\prime}\right)}$ are $K_{n, m}$-types of $\Omega(1)$. Then we define the transition coefficient $T_{\lambda, \lambda^{\prime}}$ as the composition of the following $K_{n, m}$-morphisms:

$$
T_{\lambda, \lambda^{\prime}}: \mathfrak{p}_{1} \otimes \tau_{n, m}^{\lambda} \stackrel{L}{\longrightarrow} \Omega(1) \stackrel{\mathrm{pr}}{\longrightarrow} \tau_{n, m}^{\lambda^{\prime}}
$$

where $L$ is the Lie algebra action

$$
L(X \otimes v)=X v \text { for } X \in \mathfrak{p}_{1}, v \in \tau_{n, m}^{\lambda}
$$

and pr is the projection onto the $K_{n, m^{-}}$-type $\tau_{n, m}^{\lambda^{\prime}}$.

We will call two $K_{n, m}$-types $\tau_{n, m}^{\lambda}=\tau_{n}^{\lambda} \otimes \tau_{m}^{\kappa(\lambda)}$ and $\tau_{n, m}^{\lambda^{\prime}}=\tau_{n}^{\lambda^{\prime}} \otimes \tau_{m}^{\kappa\left(\lambda^{\prime}\right)}$, (resp. two highest weights $\lambda$ and $\lambda^{\prime}$ ) neighbours if $\lambda=\lambda^{\prime} \pm \varepsilon_{i}$ for some $i$. By (13), this is equivalent to $\kappa(\lambda)=\kappa\left(\lambda^{\prime}\right) \pm \varepsilon_{j}$ for some $j$.

By Lemma 5.1.3. $\mathfrak{p}_{1} \otimes \tau_{n, m}^{\lambda}$ contains a subspace $K_{n, m}$-isomorphic to $\tau_{n, m}^{\lambda^{\prime}}$ iff $\lambda$ and $\lambda^{\prime}$ are neighbours. Hence $T_{\lambda, \lambda^{\prime}} \neq 0$ implies that $\lambda^{\prime}=\lambda \pm \varepsilon_{i}$. Given such a $\lambda^{\prime}$, the domain of $T_{\lambda, \lambda^{\prime}}$ has a unique subspace isomorphic to $\tau_{n, m}^{\lambda^{\prime}}$ and we can regard $T_{\lambda, \lambda^{\prime}}$ as a scalar multiplication by Schur's lemma. It is possible to deduce the Lie algebra action on $\Omega(1)$ from the maps $T_{\lambda, \lambda^{\prime}}$ for all $\lambda, \lambda^{\prime}$. 
5.3. Let $\mu$ denote a highest weight of $\tilde{\mathrm{O}}(m-r)$ and $v_{\mu}$ denote the highest weight of $\tau_{m-r}^{\mu}$. Then Lemma 4.2.1 states that

$$
\Omega(1, \mu)=\Omega(1)_{v_{\mu}}=\sum_{\lambda \in J}\left(\tau_{n, m}^{\lambda}\right)_{v_{\mu}}=\sum_{\lambda \in J} \tau_{n}^{\lambda} \otimes\left(\tau_{m}^{\kappa(\lambda)}\right)_{v_{\mu}}
$$

Let $\lambda^{\prime}=\lambda \pm \varepsilon_{i}$ as before and suppose $\left(\tau_{n, m}^{\lambda}\right)_{v_{\mu}}$ and $\left(\tau_{n, m}^{\lambda^{\prime}}\right)_{v_{\mu}}$ are nonzero. Let $\mathfrak{s o}(n, r)_{\mathbb{C}}=\left(\mathfrak{k}_{n, r}\right)_{\mathbb{C}} \oplus \mathfrak{p}_{2}$ denote the Cartan decomposition of the complexified Lie algebra of $\tilde{\mathrm{O}}(n, r)$. Then we define the transition coefficient $t_{\lambda, \lambda^{\prime}}$ as the composite of the following $K_{n, r}$-morphisms

$$
t_{\lambda, \lambda^{\prime}}: \mathfrak{p}_{2} \otimes\left(\tau_{n, m}^{\lambda}\right)_{v_{\mu}} \stackrel{L}{\longrightarrow} \Omega(1, \mu) \stackrel{\mathrm{pr}}{\longrightarrow}\left(\tau_{n, m}^{\lambda^{\prime}}\right)_{v_{\mu}} .
$$

Lemma 5.3.1. Suppose $\left(\tau_{n, m}^{\lambda}\right)_{v_{\mu}}$ and $\left(\tau_{n, m}^{\lambda^{\prime}}\right)_{v_{\mu}}$ are nonzero. Then $T_{\lambda, \lambda^{\prime}} \neq 0$ iff $t_{\lambda, \lambda^{\prime}} \neq 0$. Furthermore, $T_{\lambda, \lambda^{\prime}}$ and $t_{\lambda, \lambda^{\prime}}$ uniquely determine each other.

Proof. The proof follows a similar argument in $\S 6$ in [LL1].

The following lemma is very useful for later proofs.

Lemma 5.3.2. Let $1 \leq r \leq m$. Suppose $\tau_{n, m}^{\lambda}$ and $\tau_{n, m}^{\lambda^{\prime}}$ are two neighbouring $K_{n, m}$-types of $\Omega_{p}^{n, m}(1)$. Then there exists a genuine highest weight $\mu$ of $\tilde{\mathrm{O}}(m-r)$ such that $\left(\tau_{n, m}^{\lambda}\right)_{v_{\mu}}$ and $\left(\tau_{n, m}^{\lambda^{\prime}}\right)_{v_{\mu}}$ are nonzero.

Proof. This follows by applying Lemma 5.1.2 to $\tau_{m}^{\kappa(\lambda)}$ and $\tau_{m}^{\kappa\left(\lambda^{\prime}\right)}$.

Proposition 5.3.3. Given $\tilde{\mathrm{O}}(m-r)$ and suppose $\Omega_{p}^{n, m}(1)$ is nonzero. Suppose for every highest weight $\mu$ of $\tilde{\mathrm{O}}(m-r), \Omega_{p}^{n, r}(1, \mu)$ is either zero or an irreducible Harish-Chandra module of $\tilde{\mathrm{O}}(n, r)$, then $\Omega_{p}^{n, m}(1)$ is irreducible.

Proof. Suppose $\Omega(1)$ is nonzero and reducible, then by $\underline{\mathrm{Ho}}$ ], it has a nontrivial unique irreducible quotient $\theta(1)$ called the local theta lift. Let $\phi: \Omega(1) \rightarrow \theta(1)$ denote the quotient map. There exists two neighbouring $K_{n, m}$-types $\tau_{n, m}^{\lambda}$ and $\tau_{n, m}^{\lambda^{\prime}}$ such that $\phi\left(\tau_{n, m}^{\lambda}\right)$ is a $K_{n, m^{-}}$-type of $\theta(1), \tau_{n, m}^{\lambda^{\prime}}$ lies in the kernel of $\phi$, and $T_{\lambda, \lambda^{\prime}} \neq 0$. By Lemma 5.3.2, there exists a highest weight $\mu$ of $\tilde{\mathrm{O}}(m-r)$ such that $\left(\tau_{n, m}^{\lambda}\right)_{v_{\mu}}$ and $\left(\tau_{n, m}^{\lambda^{\prime}}\right)_{v_{\mu}}$ are nonzero. Then $\theta(1)_{v_{\mu}}$ contains the $K_{n, r}$-types $\left(\tau_{n, m}^{\lambda}\right)_{v_{\mu}}$ but not $\left(\tau_{n, m}^{\lambda^{\prime}}\right)_{v_{\mu}}$. Hence $\Omega(1)_{v_{\mu}}=\Omega(1, \mu)$ is reducible.

5.4. Degenerate principal series representations of $\tilde{\mathrm{O}}(n, m)$. To end this section, we will define a family of principal series representations of $\tilde{\mathrm{O}}(n, m)$ which we will need in the proofs of Theorems 1.5.1 and 1.5.2.

Assume that $n \geq m$. The group $\tilde{\mathrm{O}}(n, m)$ contains a unique parabolic subgroup $P_{m}$ with Levi decomposition $P_{m}=\left(\mathrm{GL}_{m}(\mathbb{R}) \times \tilde{\mathrm{O}}(n-m)\right) \ltimes N_{m}$.

Let $\tau_{n-m}^{\mu}$ denote a genuine representation of $\tilde{\mathrm{O}}(n-m)$. Then for $s \in \mathbb{C}$ and $\sigma=0,1$, we define $I_{n, m}\left(s, \sigma, \tau_{n-m}^{\mu}\right)$ or $I_{n, m}(s, \sigma, \mu)$ to be the (genuine) HarishChandra module of the normalized induced principal series representation

$$
\operatorname{Ind}_{P_{m}}^{\tilde{O}(n, m)} \chi_{s, \sigma} \otimes \tau_{n-m}
$$

where $\chi_{s, \sigma}$ is the one-dimensional character of $\mathrm{GL}_{m}(\mathbb{R})$ given by

$$
\chi_{s, \sigma}(g)=\left|\operatorname{det}_{m}(g)\right|^{s}\left(\operatorname{sgn}\left(\operatorname{det}_{m}(g)\right)\right)^{\sigma}, \text { for } g \in \mathrm{GL}_{m}(\mathbb{R}) .
$$


If $n=m$, then $\tau_{n-m}$ in (21) is just the nontrivial character of $\tilde{\mathrm{O}}(0)=\mathbb{Z} / 2 \mathbb{Z}$ and we write $I_{n, m}\left(s, \sigma, \tau_{n-m}\right)$ simply as $I_{n, n}(s, \sigma)$. By Frobenius reciprocity, $I_{n, n}(s, \sigma)$ has $K_{n, n}$-types

$$
I_{n, n}(s, \sigma)=\sum_{\lambda} \tau_{n}^{\lambda} \otimes\left(\tau_{n}^{\lambda} \operatorname{det}_{n}^{\sigma}\right)
$$

where the sum is taken over all genuine highest weight $\lambda$ of $\tilde{\mathrm{O}}(n)$. Hence it is $K_{n, n}$-multiplicity free. See Theorem A.2.1 for its composition series.

We recall a fact from [LL1. Suppose $n>m$, then

$$
\operatorname{Re}_{\tilde{\mathrm{O}}(n, r) \times \tilde{\mathrm{O}}(n-r)}^{\tilde{\mathrm{O}}(n, n)} I_{n, n}(s, 0)=\sum_{\mu} I_{n, r}(s, 0, \mu) \otimes \tau_{n-r}^{\mu}
$$

where the sum is taken over all genuine highest weights $\mu$ of $\tilde{\mathrm{O}}(n-r)$.

\section{IRREDUCIBILITy of $\Omega(1)$}

6.1. In this section, we will prove the assertion in Theorem 1.2.1 that $\Omega_{p}^{n, m}(1)$ is irreducible in Cases (I) and (III). The main idea is that we first restrict $\Omega(1)$ to $\tilde{\mathrm{O}}(n, 1) \times \tilde{\mathrm{O}}(m-1)$. We will show that $\Omega_{p}^{n, 1}(1, \mu)$ is irreducible for all highest weight $\mu$ of $\tilde{\mathrm{O}}(m-r)$ and Proposition 5.3 .3 will imply that $\Omega_{p}^{n, m}(1)$ is irreducible. We will postpone the proof of the unitarizability until 88 .

The proof that $\Omega(1, \mu)=\Omega_{p}^{n, 1}(1, \mu)$ is irreducible will occupy the rest of this section. First we note that $\mathrm{SO}_{0}(n, 1)$ embeds into $\tilde{\mathrm{O}}(n, 1)$. Hence we may consider $\Omega(1, \mu)$ as a Harish-Chandra module of $\mathrm{SO}_{0}(n, 1)$. We will describe the Langlands parameters and $K$-types of irreducible representations of $\mathrm{SO}_{0}(n, 1)$ in 36.2 . Next we compute the Langlands parameters of the irreducible subquotients of $\Omega(1, \mu)$ as a Harish-Chandra module of $\mathrm{SO}_{0}(n, 1)$. This will enable us to conclude that in all except one case $\Omega(1, \mu)$ is already irreducible as a $(\mathfrak{s o}(n, 1), \mathrm{SO}(n))$-module. In the exceptional case, $\Omega(1, \mu)$ is a discrete series representation of $\tilde{\mathrm{O}}(n, 1)$.

6.2. Langlands subrepresentations of $\mathrm{SO}_{0}(n, 1)$. Up to conjugation, $\mathrm{SO}_{0}(n, 1)$ has a unique parabolic subgroup with Levi decomposition $\left(\mathbb{R}^{+} \times \mathrm{SO}(n-1)\right) \ltimes N_{1}$. Set $r_{1}=\left[\frac{n-1}{2}\right]$ and let $I_{n, 1}^{0}\left(s, \tau_{\mathrm{SO}(n-1)}^{\nu}\right)$ or $I_{n, 1}^{0}(s, \nu)$ denote the Harish-Chandra module of the normalized induced principal series representation

$$
\operatorname{Ind}_{\left(\mathbb{R}^{+} \times \mathrm{SO}(n-1)\right) \ltimes N_{1}}^{\mathrm{SO}_{0}(n, 1)} \operatorname{det}_{1}^{s} \otimes \tau_{\mathrm{SO}(n-1)}^{\nu}
$$

where $\nu=\left(\nu_{1}, \ldots, \nu_{r_{1}}\right) \in \Lambda\left(r_{1}\right)$ is a highest weight of $\mathrm{SO}(n-1)$ and $s \in \mathbb{C}$. It has infinitesimal character $\left(s, \nu+\rho_{n-1}\right)$ where $\rho_{n-1} \in \frac{1}{2} \Lambda\left(r_{1}\right)$ is the half sum of all the positive roots of $\mathrm{SO}(n-1)$.

We will review Theorems 10 and 11 in $\left[\mathrm{KG}\right.$. on $I_{n, 1}^{0}(s, \nu)$ which were first stated in [Hi]. Also see [LL1. The principal series $I_{n, 1}^{0}(s, \nu)$ is $K$-multiplicity free where $K=\mathrm{SO}(n)$. It is reducible only if $s+\frac{n-1}{2} \in \mathbb{Z}$.

From now on we assume that $s+\frac{n-1}{2} \in \mathbb{Z}$ and $s \leq 0$. Let $r^{\prime}=[n / 2]$ and set $\nu_{0}=\infty$ and $\nu_{j}=0$ if $j>r_{1}$. Let $j$ denote the unique integer such that $1 \leq j \leq r^{\prime}$ and $\nu_{j-1} \geq-s-\frac{n-1}{2}+j-1 \geq \nu_{j}$. Then there exists a unique irreducible submodule $\bar{I}_{n, 1}^{0}(s, \nu)$ of $I_{n, 1}^{0}(s, \nu)$ whose sum of $K$-types $(K=\mathrm{SO}(n))$ is the sum over $\mathrm{SO}(n)$ types with highest weight $\lambda=\left(\lambda_{1}, \ldots, \lambda_{r^{\prime}}\right) \in \Lambda\left(r^{\prime}\right)$ satisfying

$$
\lambda_{i} \geq \nu_{i} \geq\left|\lambda_{i+1}\right| \text { for } i=1, \ldots, r^{\prime}
$$

and $-s-\frac{n-1}{2}+j-1 \geq\left|\lambda_{j}\right|$. 
The $\mathrm{SO}_{0}(n, 1)$ exhibits the discrete series if and only if $n=2 r^{\prime}$ is even. A discrete series representation appears as a quotient of (24) if and only if $j=r^{\prime}$ and $-s-\frac{1}{2} \geq 0$. In this case the quotient $I_{n, 1}^{0}(s, \nu) / \bar{I}_{n, 1}^{0}(s, \nu)$ is a direct sum of two discrete series representation $W^{+} \oplus W^{-}$. The sum of $K$-types of $W^{ \pm}$is the sum of $\mathrm{SO}(n)$-types of highest weight $\lambda \in \Lambda\left(r^{\prime}\right)$ such that

$$
\lambda_{1} \geq \nu_{1} \geq \ldots \geq \nu_{r^{\prime}-1} \geq\left( \pm \lambda_{r^{\prime}}\right) \geq-s+\frac{1}{2}>0 .
$$

By the formulation of Langlands parameters the above exhausts all the irreducible Harish-Chandra modules of $\mathrm{SO}_{0}(n, 1)$ and the representations are not equivalent to each other. Furthermore, $\bar{I}_{n, 1}^{0}(s, \mu)$ extends to an irreducible representation of $\mathrm{SO}(n, 1)$. The direct sum $W^{+} \oplus W^{-}$is an irreducible discrete series representation of $\mathrm{SO}(n, 1)$.

From the above description, it not difficult to show that an irreducible HarishChandra module of $\mathrm{SO}_{0}(n, 1)$ is uniquely determined by its $\mathrm{SO}(n)$-types and infinitesimal character.

Finally, we relate $I_{n, 1}(s, \sigma, \mu)$ defined in 95.4 As a $(\mathfrak{s o}(n, 1), \mathrm{SO}(n))$-module, we have

$$
I_{n, 1}\left(s, \sigma, \tau_{n-m}^{\mu}\right)=I_{n, 1}^{0}\left(s, \operatorname{Res}_{\mathrm{SO}(n-1)} \tau_{n-m}^{\mu}\right) .
$$

Similarly, an irreducible Harish-Chandra module of $\tilde{\mathrm{O}}(n, 1)$ is uniquely determined by its $K_{n, 1}$-types and infinitesimal character.

6.3. In this subsection, we will prove that $\Omega_{p}^{n, n+2}(1)$ is irreducible in Case (III). In Case (III), the $K_{n, m}$-types of $\Omega_{p}^{n, n+2}(1)$ are

$$
\sum(\overbrace{a_{1}, \ldots, a_{n-p}, 1, \ldots, 1}^{p}, 0, \ldots, 0)_{n} \otimes\left(a_{1}^{\prime}, \ldots, a_{n-p}^{\prime}, 0, \ldots, 0\right)_{m}
$$

where $a_{i}=a_{i}^{\prime}+1$ and the sum is taken over all positive integers $a_{1} \geq a_{2} \geq$ $\ldots \geq a_{n-p} \geq 1$. Note that if $n=p$, then $J_{I I I}=\left\{(1, \ldots, 1)_{n}\right\}$ (see (14)) and $\Omega_{p}^{n, n+2}(1)=\left(\operatorname{det}_{n} \otimes \mathbb{C}\right) \xi_{n, n+2}$ is a one-dimensional character of $\tilde{\mathrm{O}}(n) \times \tilde{\mathrm{O}}(n+2)$.

Let $\mu$ be a highest weight of $\tilde{\mathrm{O}}(m-r)=\tilde{\mathrm{O}}(n+1)$, then $\left(\Omega_{p}^{n, n+2}(1)\right)_{v_{\mu}}=\Omega(1, \mu)$ is nonzero if and only if $\mu$ is of the form

$$
\mu=\left(\mu_{1}, \ldots, \mu_{n-p}, 0, \ldots, 0\right)
$$

where $\mu_{i}$ are nonnegative integers. In this case $\Omega(1, \mu)$ has $K_{n, 1}$-types

$$
\Omega(1, \mu)=\sum_{\lambda} \tau_{n}^{\lambda} \otimes \xi_{1} \operatorname{det}_{1}^{\epsilon(\lambda)}
$$

where the sum is taken over all $\lambda=\left(a_{1}, \ldots, a_{n-p}, 1, \ldots, 1,0, \ldots, 0\right)$ such that $a_{i} \geq$ $\mu_{i}+1 \geq a_{i+1}$ for $i=1, \ldots, n-p$ and $\epsilon(\lambda) \equiv(n-p)+\sum_{j=1}^{n-p}\left(a_{j}-\mu_{j}\right)(\bmod 2)$.

The Lie algebras of $\tilde{\mathrm{O}}(n+1)$ and $\tilde{\mathrm{O}}(n, 1)$ are real forms of $\mathfrak{o}(n+1, \mathbb{C})$. Let $\rho_{k}=$ $\left(\frac{k}{2}-1, \frac{k}{2}-2, \ldots\right) \in \frac{1}{2} \Lambda([k / 2])$ denote the half sum of the positive roots of $\mathfrak{o}(k)$. Since $\tau_{n+1}^{\mu}$ and $\Omega(1, \mu)$ both correspond to $\Theta(\mu)$, by the correspondence of infinitesimal characters [Ho1], $\mathrm{Pz}$, both $\tau_{n+1}^{\mu}$ and $\Omega(1, \mu)$ have the same infinitesimal character $\mu+\rho_{n+1}$. Up to the action of a Weyl group element, the infinitesimal character is

$$
\left(\frac{n+1}{2}-p, \bar{\mu}+\rho_{n-1}\right) \in \frac{1}{2} \Lambda\left(\left[\frac{n+1}{2}\right]\right)
$$


where $\bar{\mu}=\left(\mu_{1}+1, \ldots, \mu_{n-p}+1,0, \ldots, 0\right) \in \frac{1}{2} \Lambda\left(\left[\frac{n-1}{2}\right]\right)$. We remark that $\bar{\mu}$ is well defined because $n-p \leq\left[\frac{n-1}{2}\right]$.

Proposition 6.3.1. Suppose we are in Case (III) and $\mu$ as in (26).

(i) As a Harish-Chandra module of $\mathrm{SO}_{0}(n, 1), \Omega(1, \mu)=\bar{I}_{n, 1}^{0}\left(\frac{n+1}{2}-p, \nu\right)$ where $\nu=\left(\mu_{1}+1, \ldots, \mu_{n-p}+1,0, \ldots, 0\right) \in \Lambda\left(\left[\frac{n-1}{2}\right]\right)$.

(ii) The Howe quotient $\Omega(1, \mu)$ is an irreducible and unitarizable Harish-Chandra module of $\tilde{\mathrm{O}}(n, 1)$.

Proof. (i) Let $V$ denote the irreducible subquotient of $\Omega(1, \mu)$ generated by the lowest $\mathrm{SO}(n)$-types. Part (i) follows by matching the possible $\mathrm{SO}(n)$-types and infinitesimal character of $V$ with the Harish-Chandra modules described in $\$ 6.2$. We will leave the details to the reader.

(ii) By Theorem 13, [KG] or [LL1, $\Omega(s, \mu)=\bar{I}_{n, 1}^{0}\left(\frac{n+1}{2}-p, \nu\right)$ is unitarizable as a Harish-Chandra module of $\mathrm{SO}_{0}(n, 1)$. By (25) an irreducible $K_{n, 1}$-type of $\Omega(1, \mu)$ is also an irreducible $\mathrm{SO}(n)$-type. Hence the restriction of the invariant Hermitian form to an irreducible $\mathrm{SO}(n)$-type is $K_{n, 1}$-invariant. This shows that the $(\mathfrak{s o}(n, 1), \mathrm{SO}(n))$-invariant Hermitian form on $\Omega(1, \mu)$ is actually $\left(\mathfrak{s o}(n, 1), K_{n, 1}\right)$ invariant.

6.4. We will now show that $\Omega(1)$ is irreducible in Case (III) in Theorem 1.2.1. By the above proposition, $\Omega(1, \mu)$ is irreducible and by Proposition $5.3 .3, \Omega_{p}^{n, n+2}(1)$ is irreducible in Case (III).

Using Vo2 and Proposition 6.3.1, one can further show that $\Omega(1, \mu)$ is the Langlands subrepresentation of $I_{n, 1}\left(s, 1, \nu^{\prime}\right)$ (see $\$ 5.4$ ) where $s=\frac{n+1}{2}-p \leq 0$ and $\nu^{\prime}=\left(\mu_{1}+1, \ldots, \mu_{n-p}+1,0, \ldots, 0\right) \in \Lambda^{+}(n-1)$.

6.5. Stable range. By Theorem 2.5.1 $\Omega_{p}^{n, m}(1)$ is irreducible in Case (I). We will sketch an alternative proof using the method similar to Case (III) above. It will yield additional information on the Langlands parameters of $\Omega_{p}^{n, 1}(1, \mu)$.

We assume that $2 p \leq n \leq m$ and $2 p<m$. Let $\mu=\left(\mu_{1}, \ldots, \mu_{[(m-1) / 2]}\right)$ be a highest weight of $\tilde{\mathrm{O}}(m-1)$. Note that $p \leq\left[\frac{m-1}{2}\right]$. Set $m^{\prime}:=\frac{m-n}{2}$. Then $\Omega(1, \mu)=\left(\Omega_{p}^{n, m}\right)_{v_{\mu}} \neq 0$ iff $\mu_{p+1}=0$ and in this case $\Omega(1, \mu)$ has $\tilde{\mathrm{O}}(n)$-types $\sum_{\lambda} \tau_{n}^{\lambda}$ where $\lambda_{p+1}=0$ and

$$
\lambda_{1} \geq \mu_{1}+m^{\prime} \geq \lambda_{2} \geq \ldots \geq \mu_{p}+m^{\prime} \geq 0 .
$$

It has infinitesimal character $\left(s, \nu+\rho_{n-1}\right)$ where

$$
s=p-\frac{n-1}{2} \text { and } \nu=\left(\mu_{1}+m^{\prime}, \ldots, \mu_{p}+m^{\prime}, 0, \ldots, 0\right) \in \Lambda\left(\left[\frac{n-1}{2}\right]\right) .
$$

A similar consideration of $\mathrm{SO}(n)$-types, $K_{n, 1}$-types and infinitesimal characters gives the following conclusions:

(i) If $n>2 p, \Omega(1, \mu)=\bar{I}_{n, 1}^{0}(s, \nu)$ is irreducible and unitarizable as a HarishChandra module of $\mathrm{SO}_{0}(n, 1)$ where $s$ and $\nu$ are given in (28). It embeds as an irreducible and unitarizable Harish-Chandra submodule of $\tilde{\mathrm{O}}(n, 1)$ into $I_{n, 1}\left(s, 0, \nu^{\prime}\right)$ (see 55.4) where $\nu^{\prime}=\left(\mu_{1}+m^{\prime}, \ldots, \mu_{p}+m^{\prime}, 0, \ldots, 0\right) \in$ $\Lambda^{+}(n-1)$.

(ii) If $n=2 p$, then $\Omega(1, \mu)$ is the Harish-Chandra module of an irreducible discrete series representation of $\tilde{\mathrm{O}}(n, 1)$.

Applying (i) and (ii) above to Proposition 5.3 .3 shows that $\Omega_{p}^{n, m}(1)$ is irreducible. 


\section{Proofs of Theorems 1.5.1 and 1.5 .2}

7.1. In this section we will proof Theorem 1.5.1 and some cases of Theorem 1.5.2 First suppose $\mu=\left(\mu_{1}, \ldots, \mu_{k}, 0, \ldots, 0\right)_{m-r}$ where $\mu_{k} \geq 1$ and $k \leq p$. We will compute the infinitesimal character of $\Omega(1, \mu)$ using the correspondences of infinitesimal characters [Ho1, $\mathrm{Pz}$. If $k>\left[\frac{n+r}{2}\right]$ and $m=n$ (part of Case (II)), then $\mu_{m-r-k+1}=\ldots=\mu_{k}=1$ and $\Omega(1, \mu)$ has infinitesimal character

$$
\left(\mu_{1}, \ldots, \mu_{n+r-k+1}, 0, \ldots, 0\right)+\rho_{n+r} \in \frac{1}{2} \Lambda\left(\left[\frac{n+r}{2}\right]\right)
$$

where $\rho_{n+r}$ is the half-sum of the positive roots of $\mathrm{SO}(n+r)$. Otherwise, if $k \leq\left[\frac{n+r}{2}\right]$ or we are in Case (III), then the infinitesimal character is

$$
\left(\mu_{1}, \ldots, \mu_{k}, 0, \ldots, 0\right)+\left(\frac{m-n-2 r}{2} \mathbf{1}_{p}, \mathbf{0}_{\left[\frac{n+r-2 p}{2}\right]}\right)+\rho_{n+r} \in \frac{1}{2} \Lambda\left(\left[\frac{n+r}{2}\right]\right) .
$$

Lemma 7.1.1. The Howe quotients $\Omega(1, \mu)$ have distinct infinitesimal characters for distinct genuine highest weights $\mu$ of $\tilde{\mathrm{O}}(m-r)$.

Proof. We observe this from the above description. Nevertheless, we will give an alternative proof. If $\Omega(1, \mu)$ and $\Omega\left(\chi, \mu^{\prime}\right)$ have the same infinitesimal character, then by the correspondence of infinitesimal characters, $\Theta(\mu)$ and $\Theta\left(\mu^{\prime}\right)$ have the same infinitesimal character. Again by the correspondence, $\tau_{n-r}^{\mu}$ and $\tau_{n-r}^{\mu^{\prime}}$ have the same infinitesimal character. This means $\mu=\mu^{\prime}$.

7.2. Case $\mathbf{I}$ in Theorem 1.2.1. Suppose we are in the stable range and the dual pair is not $(\widetilde{\mathrm{Sp}}(p, \mathbb{R}), \widetilde{\mathrm{O}}(2 p, 2 p))$, then Theorem 2.5.1 is already established, and Theorems 1.5.1 and 1.5.2 now follow from Corollary 4.3.1

7.3. Case II in Theorem 1.2.1, Next we suppose $n=m$. We do not assume that it is outside stable range. By [Zhu (see (37)), $\Omega(1)$ is a submodule of the following degenerate principal series representation of $\tilde{\mathrm{O}}(n, n)$ (see $\$ 5.4$ )

$$
I_{n, n}\left(p-\frac{n-1}{2}, 0\right)
$$

By comparing (23) and (1) we obtain the following proposition.

Proposition 7.3.1. The Howe quotient $\Omega(1, \mu)$ embeds into $I_{n, r}\left(p-\frac{n-1}{2}, 0, \mu\right)$.

By the above proposition, Theorems 1.5 .1 and 1.5 .2 will follow from the next theorem.

Theorem 7.3.2. Suppose we are given $\tilde{\mathrm{O}}(n, r) \times{ }_{2} \tilde{\mathrm{O}}(n-r) \subset \tilde{\mathrm{O}}(n, n)$ as in 42.2 . Let $\tilde{\Omega}$ denote an irreducible subquotient of the degenerate principal series representations of $\tilde{\mathrm{O}}(n, n)$ in (30).

(i) For every genuine highest weight $\mu$ of $\tilde{\mathrm{O}}(n-r),(\tilde{\Omega})_{v_{\mu}}$ is either zero or an irreducible subquotient of $I_{n, r}\left(p-\frac{n-1}{2}, 0, \mu\right)$.

(ii) The subquotient $\tilde{\Omega}$ is unitarizable iff $(\tilde{\Omega})_{v_{\mu}}$ is unitarizable for every highest weight $\mu$ of $\tilde{\mathrm{O}}(n-r)$.

Proof. We have proven a corresponding statement for $\mathrm{SO}_{0}(n, n)$ in Part 2 of [LL1]. The extension to $\tilde{\mathrm{O}}(n, n)$ is a straightforward exercise after taking into account Theorem A.2.1 and (23). We will leave the details to the reader.

The following lemma is useful in the next section. 
Lemma 7.3.3. Let $\tilde{\Omega}$ be an irreducible subquotient of $\Omega(1)$, then $T_{\lambda, \lambda^{\prime}}$ defined in (20) is nonzero for any two neighbouring $K_{n, m}$-types $\tau_{n, m}^{\lambda}$ and $\tau_{n, m}^{\lambda^{\prime}}$ in $\tilde{\Omega}$.

Proof. If $n=m$, the lemma follows from the computation of the transition coefficients of the degenerate principal series in (30) due to [J], [Le], [Sa] and Zha]. Suppose $n<m$. By Lemma 5.3.2, there exists highest $\mu$ of $\tilde{\mathrm{O}}(m-1)$ such that $\left(\tau_{n, m}^{\lambda}\right)_{v_{\mu}}$ and $\left(\tau_{n, m}^{\lambda^{\prime}}\right)_{v_{\mu}}$ are nonzero $K_{n, 1}$-types of $\tilde{\Omega}_{v_{\mu}}$. Since $\tilde{\Omega}_{v_{\mu}}$ is an irreducible Harish-Chandra module of $\tilde{\mathrm{O}}(n, 1)$, it follows from [Hi], [KG] or [LL1] that $t_{\lambda, \lambda^{\prime}} \neq 0$. By Lemma $5.3 .1 T_{\lambda, \lambda^{\prime}} \neq 0$ also.

\section{UNITARITY}

8.1. In this section let $\tilde{\Omega}$ denote an irreducible subquotient of $\Omega_{p}^{n, m}(1)$. Our goal is to complete the proof of Theorem 1.5.2. First we will show that $\tilde{\Omega}$ exhibits a (not necessary positive definite) Hermitian product $H$. Next we will prove the remaining cases of Theorem 1.5.2 with the help of the Hermitian form. Finally, in 88.8 we will determine the unitarizability of all the irreducible subquotients of $\Omega(1, \mu)$.

8.2. Let $\tilde{\Omega}^{\mathrm{h}}$ denote the Hermitian dual representation of $\tilde{\Omega}$.

Proposition 8.2.1. $\quad$ (i) The $\left(\mathfrak{s o}(n, m), K_{n, m}\right)$-modules $\tilde{\Omega}$ and $\tilde{\Omega}^{\mathrm{h}}$ are isomorphic irreducible modules. Hence $\tilde{\Omega}$ exhibits a nondegenerate invariant Hermitian form.

(ii) Let $\mu$ denote a genuine highest weight of $\tilde{\mathrm{O}}(m-r)$. Then the restriction of the invariant Hermitian form to $\tilde{\Omega}_{v_{\mu}}$ is nondegenerate.

First we see that (i) implies (ii). Indeed let $\tau_{n, m}^{\lambda}$ be a $K_{n, m}$-type of $\tilde{\Omega}$. Since $\tilde{\Omega}$ is $K_{n, m}$-multiplicity free, the invariant Hermitian form on $\tilde{\Omega}$ in (i) is either positive or negative definite on $\tau_{n, m}^{\lambda}$. Hence the restriction to $\left(\tau_{n, m}^{\lambda}\right)_{v_{\mu}}$ is also positive or negative definite. This proves (ii).

8.3. It remains to prove Proposition 8.2.1(i). If we are in the stable range, then $\Omega(1)$ is irreducible and unitarizable and the above proposition holds. If $n=m$, then $\tilde{\Omega}$ is a subquotient of one of the degenerate principal series representations in (30) and the proposition follows from [J], [Sa] or [Zha].

From the above discussion, it remains to prove Case (III) in Theorem 1.2.1. One method is to show that $\tilde{\Omega}$ and $\tilde{\Omega}^{\mathrm{h}}$ has the same Langlands parameter. Nevertheless, we will give a more elementary proof which works for Cases I, II and III. The idea is to restrict $\Omega(1)$ to $\tilde{\mathrm{O}}(n, 1)$. The proof will occupy 8.4 and 8.5 . Readers may proceed to 8.6 without loss of continuity.

8.4. Suppose $\tilde{\Omega}$ has $K_{n, m}$-types (see (14))

$$
\tilde{\Omega}=\sum_{\lambda \in I} \tau_{n, m}^{\lambda}
$$

where $I \subset J$. Then $\tilde{\Omega}^{\mathrm{h}}$ has the same $K_{n, m}$-types as $\tilde{\Omega}$ and we write

$$
\tilde{\Omega}^{\mathrm{h}}=\sum_{\lambda \in I} \hat{\tau}_{n, m}^{\lambda}
$$

and let $\phi_{\lambda}: \tau_{n, m}^{\lambda} \rightarrow \hat{\tau}_{n, m}^{\lambda}$ denote an isomorphism of irreducible $K_{n, m}$-modules. 
Suppose $\lambda, \lambda^{\prime} \in I$ are neighbours and let $T_{\lambda, \lambda^{\prime}}$ and $T_{\lambda, \lambda^{\prime}}^{\mathrm{h}}$ denote the transition coefficients of $\tilde{\Omega}$ and $\tilde{\Omega}^{\mathrm{h}}$ respectively. By Lemma 7.3.3. $T_{\lambda, \lambda^{\prime}} \neq 0$ for neighbouring $\lambda, \lambda^{\prime}$. It is easy to see that this implies $T_{\lambda, \lambda^{\prime}}^{\mathrm{h}} \neq 0$ too.

Lemma 8.4.1. There exists a nonzero complex number $C\left(\lambda, \lambda^{\prime}\right)$ such that the following diagram commutes:

$$
\begin{array}{ccc}
\mathfrak{p}_{1} \otimes \tau_{n, m}^{\lambda} & \stackrel{T_{\lambda, \lambda^{\prime}}}{\longrightarrow} & \tau_{n, m}^{\lambda^{\prime}} \\
\mathrm{id} \otimes \phi_{\lambda} \downarrow & & \downarrow C\left(\lambda, \lambda^{\prime}\right) \phi_{\lambda^{\prime}} \\
\mathfrak{p}_{1} \otimes \hat{\tau}_{n, m}^{\lambda} & \stackrel{T_{\lambda, \lambda^{\prime}}^{\mathrm{h}}}{\longrightarrow} & \hat{\tau}_{n, m}^{\lambda^{\prime}}
\end{array}
$$

Proof. Let $V_{\lambda^{\prime}}$ be the unique subspace of $\mathfrak{p}_{1} \otimes \tau_{n, m}^{\lambda}$ such that $V_{\lambda^{\prime}}$ is isomorphic to $\tau_{n, m}^{\lambda^{\prime}}$ (see 95.2$)$. The maps $T_{\lambda, \lambda^{\prime}}^{\mathrm{h}} \circ\left(\mathrm{id} \otimes \phi_{\lambda}\right)$ and $\phi_{\lambda^{\prime}} \circ T_{\lambda, \lambda^{\prime}}$ are $K_{n, m^{\prime}}$-isomorphisms from $V_{\lambda^{\prime}}$ to $\hat{\tau}_{n, m}^{\lambda^{\prime}}$. Hence by Schur's Lemma they differ by a nonzero constant $C\left(\lambda, \lambda^{\prime}\right)$.

8.5. It follows that if $C\left(\lambda, \lambda^{\prime}\right)=1$ for all $\lambda$ and $\lambda^{\prime}$ in Lemma 8.4.1, then $\sum_{\lambda \in I} \phi_{\lambda}$ is a $\left(\mathfrak{s o}(n, m), K_{n, m}\right)$-isomorphism from $\tilde{\Omega}$ to $\tilde{\Omega}^{\mathrm{h}}$. More generally, if there exists $B(\lambda) \in \mathbb{C}^{*}$ such that $C\left(\lambda, \lambda^{\prime}\right)=B\left(\lambda^{\prime}\right) B(\lambda)^{-1}$, then $\sum_{\lambda \in I} B(\lambda) \phi_{\lambda}$ is a $\left(\mathfrak{s o}(n, m), K_{n, m}\right)$ isomorphism from $\tilde{\Omega}$ to $\tilde{\Omega}^{\mathrm{h}}$. Hence our goal is to replace $\phi_{\lambda}$ by a nonzero multiple of itself so that $C\left(\lambda, \lambda^{\prime}\right)=1$ for all $\lambda, \lambda^{\prime}=\lambda \pm \varepsilon_{i}$.

One way to achieve the above is as follows: First we set $r=1$ and we consider the dual pair $(\tilde{\mathrm{O}}(n, 1), \tilde{\mathrm{O}}(m-1))$. By Lemma 5.3.2 there exists a highest weight $\mu$ of $\tilde{O}(m-1)$ such that $\left(\tau_{n, m}^{\lambda}\right)_{v_{\mu}}$ and $\left(\tau_{n, m}^{\lambda^{\prime}}\right)_{v_{\mu}}$ are nonzero $K_{n, 1}$-types of $\tilde{\Omega}_{v_{\mu}}$. Since $\tilde{\Omega}_{v_{\mu}}$ is irreducible and it has the same $K_{n, 1}$-types and infinitesimal character as $\tilde{\Omega}_{v_{\mu}}^{\mathrm{h}}$, by $\varangle 6.2$ they are isomorphic as $\left(\mathfrak{s o}(n, 1), K_{n, 1}\right)$-modules. Let

$$
\phi(\mu): \tilde{\Omega}_{v_{\mu}} \rightarrow \tilde{\Omega}_{v_{\mu}}^{\mathrm{h}}
$$

denote the isomorphism between them. Since the isomorphism is unique up to a nonzero scalar, we may assume that $\phi(\mu)=\phi_{\lambda}$ on $\left(\tau_{n, m}^{\lambda}\right)_{v_{\mu}}$. We scale $\phi_{\lambda^{\prime}}$ so that $\phi(\mu)=\phi_{\lambda^{\prime}}$ on $\left(\tau_{n, m}^{\lambda^{\prime}}\right)_{v_{\mu}}$. It is now clear that $C\left(\lambda, \lambda^{\prime}\right)=C\left(\lambda^{\prime}, \lambda\right)=1$.

We claim that the scaling of $\phi_{\lambda^{\prime}}$ is independent of $\mu$. Indeed, first we may assume that $C\left(\lambda, \lambda^{\prime}\right)=1$ after scaling using $\mu$. Let $\beta$ be another highest weight of $\tilde{\mathrm{O}}(m-r)$. Let $\mathfrak{s o}(n, 1)_{\mathbb{C}}=\left(\mathfrak{k}_{n, 1}\right)_{\mathbb{C}} \oplus \mathfrak{p}_{2}$ denote the Cartan decomposition and let $t_{\lambda, \lambda^{\prime}}$ and $t_{\lambda, \lambda^{\prime}}^{\mathrm{h}}$ denote the transition coefficients of $\tilde{\Omega}_{v_{\beta}}$ and $\tilde{\Omega}_{v_{\beta}}^{\mathrm{h}}$ respectively. Then in the commutative diagram in Lemma 8.4.1. $C\left(\lambda, \lambda^{\prime}\right)=1$ so $t_{\lambda, \lambda^{\prime}}^{\mathrm{h}} \circ \phi_{\lambda}=\phi_{\lambda^{\prime}} \circ t_{\lambda, \lambda^{\prime}}$ on $\mathfrak{p}_{2} \otimes\left(\tau_{n, m}^{\lambda}\right)_{v_{\beta}}$. This proves our claim.

From now on, it is a matter of bookkeeping to make sure that we can scale all the $\phi_{\lambda}$ inductively in a uniform manner. First for $\lambda=\left(\lambda_{1}, \ldots, \lambda_{n}\right) \in I$ in (31) we define $|\lambda|=\sum_{i} \lambda_{i}$. Let $\lambda_{0}$ denote the $\lambda$ in $I$ with the smallest $|\lambda|$. From the description of the $K_{n, m}$-types of irreducible subquotient $\Omega(1)$ in (15) to (17) or Theorem A.2.2, $\lambda_{0}$ is unique. Starting with the $\phi_{\lambda_{0}}$, we scale $\phi_{\lambda}$ using the method above inductively on $|\lambda|$. We will leave the details to the reader. This proves Proposition 8.2.1.

8.6. We will now prove that $\Omega(1)$ is unitarizable in Case (III) in Theorem 1.2.1. Indeed, let $H$ denote the (unique up to scalar) $\mathfrak{s o}(n, m)$-invariant Hermitian form on $\Omega(1)$ as given in Proposition 8.2.1(i). 
Suppose $H$ does not have positive signature, then there exists two nonzero $K_{n, 1^{-}}$ types $\tau_{n, m}^{\lambda}$ and $\tau_{n, m}^{\lambda+\epsilon_{i}}$ of $\Omega(1)$ where $H$ takes opposite signatures. By Lemma 5.3 .2 there exists a genuine highest weight $\mu$ of $\tilde{\mathrm{O}}(m-1)$ such that $\left(\tau_{n, m}^{\lambda}\right)_{v_{\mu}}$ and $\left(\tau_{n, m}^{\lambda+\epsilon_{i}}\right)_{v_{\mu}}$ are nonzero. Hence $H^{\prime}$ have opposite signatures on these two $K_{n, 1}$-types of $\Omega(1)_{v_{\mu}}$.

On the other hand, by Proposition 6.3.1, $\Omega(1)_{v_{\mu}}$ is irreducible and unitarizable. Therefore, $H^{\prime}$ is the unique Hermitian form on $\Omega(1)_{v_{\mu}}$ and it is positive definite. This leads to a contradiction and we conclude that $H$ is positive definite.

8.7. We will now prove Theorems 1.5.1 and 1.5 .2 for $\Omega(1)$ in Case (III). Indeed, this follows from Corollary 4.3 .1 because $\Omega(1)$ is irreducible and unitarizable by \$6.4 and 8.6 .

We would like to mention that up to this point we have completed the proofs of Theorems 1.2.1, 1.5.1 and 1.5.2.

8.8. Composition series of $\Omega_{p}^{n, r}(1, \mu)$. We will describe the composition series of $\Omega(1, \mu)$ and its unitarizable irreducible subquotients.

Let $q=m-r$ and let $\mu=\left(\mu_{1}, \ldots, \mu_{q}\right)$ be a highest weight of $\tilde{\mathrm{O}}(q)$. We extend $\mu$ such that $\mu_{i}=\infty$ for $i \leq 0$ and $\mu_{i}=0$ for $i>q$.

Theorem 8.8.1. The Howe quotient $\Omega_{p}^{n, r}(1, \mu)$ is nonzero if and only if one of the following situations holds:

(i) $p \leq \min (n, r+q), \mu_{p+1}=0$ and $\mu_{p-q} \geq \max \left(0, \frac{n-r-q}{2}\right)$.

(ii) $p \leq n \leq 2 p-1, r+q=n+2$ and $\mu_{n-p+1}=0$.

(iii) $p+2 \leq n \leq 2 p+1, r+q=n-2, \mu_{n-p+1} \leq 1, \mu_{p+1}=0$ and $\mu_{p-q} \geq 1$.

(iv) $n=r+q \leq 2 p$ and $\mu_{p+1}=0$.

In (i), (ii) and (iii) $\Omega(1, \mu)$ is irreducible and unitarizable.

Proof. In order to determine whether $\Omega_{p}^{n, r}(1, \mu)=\left(\Omega_{p}^{n, r+q}\right)(1)_{v_{\mu}}$ is nonzero, we apply the branching rules to the $K_{n, m}$-types of $\Omega_{p}^{n, r+q}(1)$ in four situations. These four situations are (i) Case I; (ii) Case III where $r+q=n+2$; (iii) Case III where $r+q=n-2$ and (iv) Case II. The computations are straightforward so we will leave them to the reader.

Suppose $n=r+q \leq 2 p$ and $\mu_{p+1}=0$ so that $\Omega(1, \mu) \neq 0$ as in Theorem 8.8.1(iv). Set $i_{0}=\max \left\{i: \mu_{i} \geq p-n+1+i\right\}$ and $j_{0}=\max \left\{j: \mu_{j-r} \geq p-n+1+j\right\}$. Note that $1 \leq i_{0} \leq j_{0} \leq n / 2$. We will describe the composition series of $\Omega_{p}^{n, r}(1, \mu)$ and the unitary of its subquotients with the help of Theorem A.2.2. Also see the appendix for the notation $R(n, s, 0, i)$.

Theorem 8.8.2. Suppose $n=r+q \leq 2 p, s=p-\frac{n-1}{2}$ and $\mu_{p+1}=0$.

(i) The Howe quotient $\Omega_{p}^{n, r}(1, \mu)$ is a submodule of $I_{n, r}(s, 0, \mu)$ (see \$5.4). It has a filtration of length $j_{0}-i_{0}+1$. In particular, $\Omega_{p}^{n, r}(1, \mu)$ is irreducible if and only if $i_{0}=j_{0}$.

(ii) Suppose $\frac{n}{2} \leq p \leq n-1$ and $\mu_{n-p}=0$, then $i_{0}=0$ and by Theorem $\mathrm{A.2.2}$ (ii) $\Omega_{p}^{n, r}(1, \mu)=\left(\Omega_{p}^{n, n}(1)\right)_{v_{\mu}}$ has module diagram

$$
\left(R^{+}(n, s, 0)\right)_{v_{\mu}} \rightarrow(R(n, s, 0, n-p))_{v_{\mu}} \rightarrow \cdots \rightarrow\left(R\left(n, s, 0, j_{0}\right)\right)_{v_{\mu}}
$$

In this case the theta lift $\left(R^{+}(n, s, 0)\right)_{v_{\mu}}$ is unitarizable and it is the dual representation of the Howe quotient $\Omega_{n-1-p}^{n, r}(1, \mu)$ lifted from the group $\widetilde{\mathrm{Sp}}(n-1-p, \mathbb{R})$ in Theorem 8.8.1(i). 
(iii) Suppose $\frac{n}{2} \leq p \leq n-1$ and $\mu_{n-p}>0$, or, $n \leq p$, then by Theorem A.2.2(ii)(iii) $\Omega(1, \mu)$ has module diagram

$$
\left.\left(R\left(n, s, 0, i_{0}\right)\right)_{v_{\mu}} \rightarrow\left(R\left(n, s, 0, i_{0}+1\right)\right)_{v_{\mu}} \rightarrow \cdots \rightarrow\left(R\left(n, s, 0, j_{0}\right)\right)\right)_{v_{\mu}} .
$$

An irreducible subquotient in (ii) and (iii) is unitarizable if and only if it is in one of the following situations:

(a) The subquotient $\left(R^{+}(n, s, 0)\right)_{v_{\mu}}$ in (ii).

(b) When $n$ is even, $j_{0}=n / 2$, that is, $\mu_{q-n / 2} \geq p+1-n / 2$. Then $(R(n, s, 0$, $n / 2))$ is unitarizable and therefore $(R(n, s, 0, n / 2))_{v_{\mu}}$ is unitarizable too.

(c) Up to tensoring $\tau_{q}^{\mu}$ with $\operatorname{det}_{\mathrm{O}(q)}, \mu_{j_{0}-r+1}=0$. Then $\left(R\left(n, s, 0, j_{0}\right)\right)_{v_{\mu}}$ is unitarizable.

In (b) and (c) above $\left(R\left(n, s, 0, j_{0}\right)\right)_{v_{\mu}}$ is not the theta lift because $i_{0}<j_{0}$. Hence we conclude that in Case (II), the theta lift is unitarizable if and only if it is $\left(R^{+}(n, s, 0)\right)_{v_{\mu}}$ in (ii) above.

Proof. We will briefly explain the proof of the above theorem. In order to determine which subquotients of $\Omega(1, \mu)=\Omega(1)_{v_{\mu}}$ are nonzero, we follow the method of proof in Theorem 8.8 .1 by applying the branching rules to the $K_{n, n}$-types of the subquotients of $\Omega(1)$. The computation is a little tedious but straightforward, so we will leave them to the reader.

In (ii), $R^{+}$and $\Omega(1, \mu)$ are dual representations because they have the same $K_{n, r^{-}}$-types and they lie in $I_{n, n}(s, 0, \mu)$ and $I_{n, n}(-s, 0, \mu)$ respectively.

Parts (a) and (b) are self-explanatory. For the unitarizability of the remaining subquotients, we use Theorem 7.3 .2 (b). First we restrict a subquotient to $\mathfrak{s o}(n, 1)$ and check this against the list of unitarizable Harish-Chandra modules of $\tilde{\mathrm{O}}(n, 1)$. This is a very tedious case-by-case consideration and we will not do it here.

8.9. Associated Varieties. It is interesting to compute the associated varieties and associated cycles of $\Omega(1)$ and $\Omega(1, \mu)$. See, for example, Vo4 for the definition of associated variety and associated cycle. Some of the results in this area are HL, NZ1] and [NZ2]. There are also some nice relationships between discretely decomposable restrictions and associated varieties $\mathrm{Ko} 2$. Applying Theorem 3.7 in Ko2 to Theorem 1.5.1 gives the following proposition.

Proposition 8.9.1. Let $\tilde{\Omega}$ be an irreducible subquotient of $\Omega(1)$ and let $\mu$ and $\delta$ be two genuine highest weights of $\tilde{\mathrm{O}}(m-r)$. Suppose $\tilde{\Omega}_{v_{\mu}}$ and $\tilde{\Omega}_{v_{\delta}}$ are nonzero, then they have the same associated variety.

\section{Relations with Vogan-Zuckerman's $A_{\mathfrak{q}}(\lambda)$}

9.1. Recall that $\Omega_{p}^{n, m}(1)$ (resp. $\Omega_{p}^{n, r}(1, \mu)$ ) has a unique irreducible quotient called the theta lift. We would like to relate unitarizable theta lifts in the last section with Vogan-Zuckerman's $A_{\mathfrak{q}}(\lambda)$.

First by Theorem 8.8 .2 (ii), we see that in Case (II), the unitarizable theta lifts are dual to certain theta lifts in Case (I). Therefore, we will only concentrate on Cases (I) and (III) where $\Omega(1)$ and $\Omega(1, \mu)$ are irreducible and they are the theta lifts.

Let $\mu=\left(\mu_{1}, \ldots, \mu_{k}, 0, \ldots, 0\right)_{m-r}$ be a highest weight of $\tilde{\mathrm{O}}(m-r)$. Here $\mu_{k} \geq 1$ and $k \leq p$. We include the most degenerate case where $m=r$ so that $\mu=\emptyset$. In this case we set $\Omega(1, \mu)=\Omega(1)$. 
We will assume that $\Omega(1, \mu)$ is nonzero and unitarizable in Cases (I) and (III) as described in Theorems 1.2.1 and Theorem 8.8.1. It is easier to work with $\Omega(1, \mu)$ as a Harish-Chandra module of $\mathrm{SO}(n, r)$. Unfortunately, it is not always irreducible so we will describe an irreducible Harish-Chandra submodule $\Omega^{0}(1, \mu)$ of $\Omega(1, \mu)$ below.

Using the branching rule and the $K_{n, m}$-types of $\Omega(1)$, we check that $\Omega(1, \mu)$ has lowest $K_{n, r}$-type

$$
\tau_{n, r}=\left(\left(\mu_{1}, \ldots, \mu_{k}, 0, \ldots, 0\right)_{n}+\left(\frac{m-n}{2} \mathbf{1}_{p}, \mathbf{0}_{n-p}\right)_{n}\right) \otimes \xi_{r} \operatorname{det}_{r}^{\epsilon}
$$

where $\epsilon=1$ if $n=m+2 \leq 2 p+1$ and $\epsilon=0$ if otherwise. Note that $\operatorname{SO}(n, r) \times$ $\mathbb{Z} / 2 \mathbb{Z}$ embeds into $\tilde{\mathrm{O}}(n, r)$. It is clear that the restriction of $\tau_{n, r}$ to $\mathrm{S}(\mathrm{O}(n) \times$ $\mathrm{O}(r))$ remains irreducible. Since $\mathfrak{s o}(n, r)$ acting on the lowest $K_{n, r}$-type generates the (irreducible) $\Omega(1, \mu)$, the restriction of $\Omega(1, \mu)$ as a Harish-Chandra module of $\mathrm{SO}(n, r)$ is irreducible.

Furthermore, $\tau_{n, r}$ is a reducible representation of $K_{n, r}^{0}:=\mathrm{SO}(n) \times \mathrm{SO}(r)$ if and only if $m>n=2 p$. First suppose $\tau_{n, r}$ is irreducible over $K_{n, r}^{0}$, then a similar argument shows that it is an irreducible Harish-Chandra module of $\mathrm{SO}_{0}(n, r)$. We set $\Omega^{0}(1, \mu)=\Omega(1, \mu)$ in this case.

Next, suppose $\tau_{n, r}$ is reducible over $K_{n, r}^{0}$. By (9) $\tau_{n, r}$ decomposes into a sum of two irreducible representations $\tau_{n, r}^{+} \oplus \tau_{n, r}^{-}$of $K_{n, r}^{0}$. By a result of Vogan, an irreducible Harish-Chandra module of the connected $\mathrm{SO}_{0}(n, r)$ has only one lowest $K_{n, r}^{0}$-type so $\Omega(1, \mu)$ is reducible. Let $\Omega^{0}(1, \mu)$ be the irreducible $\left(\mathfrak{s o}(n, r), K_{n, r}^{0}\right)$ module generated by $\tau_{n, r}^{+}$. A simple application of Frobenius reciprocity shows that

$$
\Omega(1, \mu)=\operatorname{Ind}_{\left(\mathfrak{s o}(n, r), K_{n, r}^{0}\right)}^{(\mathfrak{s o}(n, r), \mathrm{S}(\mathrm{O}(n) \times \mathrm{O}(r)))} \Omega^{0}(1, \mu) .
$$

9.2. Before we state the next lemma, we need to define a $\theta$-stable parabolic subalgebra $\mathfrak{q}$ of $\mathfrak{s o}(n, r)_{\mathbb{C}}$. Let $\mathfrak{h}=\mathfrak{t} \oplus \mathfrak{a}$ denote a $\theta$-stable fundamental Cartan subalgebra of $\mathfrak{s o}(n, r)_{\mathbb{C}}$. Here $\mathfrak{t}$ is a Cartan subalgebra of $K_{n, r}^{0}$. Let $p \leq\left[\frac{n}{2}\right]$ and let $(p, p-1, \ldots, 1,0, \ldots, 0)_{\left[\frac{n+r}{2}\right]}$ denote a weight of $\mathfrak{h}$. Let $\mathfrak{q}=\mathfrak{l} \oplus \mathfrak{u}$ denote the $\theta$-stable parabolic subalgebra of $\mathfrak{s o}(n, r)_{\mathbb{C}}$ defined by this weight. Note that $\mathfrak{l}$ is the complexified Lie algebra of Levi subgroup $\mathbb{T}_{p} \cdot \mathrm{SO}_{0}(n-2 p, r)$ of $\mathrm{SO}_{0}(n, r)$ where $\mathbb{T}_{p}$ is a compact torus of dimension $p$.

Let $\rho_{n+r}$ (resp. $\left.\rho(\mathfrak{u}), \rho(\mathfrak{u} \cap \mathfrak{p})\right)$ denote the half-sum of positive roots of $\mathfrak{s o}(n+r)$ (resp. $\mathfrak{u}, \mathfrak{u} \cap \mathfrak{p})$ with respect to $\mathfrak{h}$. We check that

$$
2 \rho(\mathfrak{u} \cap \mathfrak{p})=\left(r \mathbf{1}_{p}, 0, \ldots, 0\right) \in \Lambda^{+}\left(\left[\frac{n+r}{2}\right]\right) .
$$

We define $A_{\mathfrak{q}}(\lambda)$ and $A^{\mathfrak{q}}(\lambda)$ as on page 330 in $\left[\mathrm{KnV}\right.$. We note that $A_{\mathfrak{q}}(\lambda)$ and $A^{\mathfrak{q}}(\lambda)$ are isomorphic in the weakly fair range. Both have infinitesimal character $\lambda+\rho_{n+r}$ and lowest $K_{n, r}^{0}$-type $\left.(\lambda+\rho(\mathfrak{u} \cap \mathfrak{p}))\right|_{\mathfrak{t}}$. It is a well-known result of Vogan that $A_{\mathfrak{q}}(\lambda)$ is unitarizable in the weakly fair range [Vo3].

Lemma 9.2.1. The irreducible unitarizable $\left(\mathfrak{s o}(n, r), K_{n, r}^{0}\right)$-module $\Omega^{0}(1, \mu)$ has the same infinitesimal character and lowest $K_{n, r}^{0}$-type as $A_{\mathfrak{q}}(\lambda)$ (and also $A^{\mathfrak{q}}(\lambda)$ ) where $\mathfrak{q}$ is the $\theta$-stable parabolic subalgebra of $\mathfrak{s o}(n, r)_{\mathbb{C}}$ defined above and

$$
\lambda=\left(\mu_{1}, \ldots, \mu_{k}, 0, \ldots, 0\right)_{N}+\left(\frac{m-n-2 r}{2} \mathbf{1}_{p}, \mathbf{0}_{N-p}\right)_{N}
$$

where $N=\left[\frac{n+r}{2}\right]$. 
Proof. We simply compare the infinitesimal character and lowest $K_{n, r}^{0}$-type of $A_{\mathfrak{q}}(\lambda)$ with those of $\Omega(1, \mu)$ in (29) and (32).

We remark that $\lambda$ is in the fair range (resp. weakly fair range, weakly good range) if and only if $\mu_{p}>p-\frac{m-r}{2}$ (resp. $\mu_{p} \geq p-\frac{m-r}{2}, \mu_{p} \geq \frac{n-m}{2}+r-1$ ). In particular, if $r=m$, then it is never in the weakly fair range.

We recall Theorem 6.1 in $\left[\mathrm{VZ}\right.$, which gives the characterization of $A_{\mathfrak{q}}(\lambda)$. Suppose

$$
\left\langle\alpha,\left.\lambda\right|_{\mathfrak{t}}\right\rangle \geq 0 \quad \text { for all } \alpha \text { roots in } \mathfrak{u} .
$$

Then any irreducible unitarizable Harish-Chandra module having the same infinitesimal character $\lambda+\rho$ and containing the $K_{n, r}^{0}$-type $\tau_{n, r}$ in (32) is isomorphic to $A_{\mathfrak{q}}(\lambda)$. This characterizes all irreducible Harish-Chandra modules with nonzero $(\mathfrak{g}, K)$-cohomology. Combining this characterization with Lemma 9.2.1 gives the following proposition.

Proposition 9.2.2. In Lemma 9.2.1 the irreducible $\Omega(1, \mu)^{0}$ is isomorphic to $A_{\mathfrak{q}}(\lambda)$ if $2 \mu_{p}+m-n \geq 2 r \geq 2$.

If we apply the above proposition to Corollary 5.1.4, we get the following corollary.

Corollary 9.2.3. Let $N=\left[\frac{n+r}{2}\right]$ and $\lambda$ as in (33) such that $2 \mu_{p}+m-n \geq 2 r \geq 4$. Then

$$
\operatorname{Res}_{\left(\mathfrak{s o}(n, r-1), K_{n, r-1}^{0}\right)}^{\left(\mathfrak{s o}(n, r), K_{n, r}^{0}\right)} A_{\mathfrak{q}}(\lambda)=\sum_{\lambda^{\prime}} A_{\mathfrak{q}^{\prime}}\left(\lambda^{\prime}\right)
$$

where $\lambda^{\prime}=\left(\mu_{1}^{\prime}, \ldots, \mu_{k}^{\prime}, 0, \ldots, 0\right)_{N-1}+\left(\frac{m-n-2 r}{2} \mathbf{1}_{p}, \mathbf{0}_{N-1-p}\right)_{N-1}$ is taken over

$$
\mu_{1}^{\prime} \geq \mu_{1} \geq \mu_{2}^{\prime} \geq \ldots \geq \mu_{k} \geq \mu_{k}^{\prime} \geq 0 .
$$

When $n$ is even, then $A_{\mathfrak{q}}(\lambda)$ is in the discrete series and the corollary is a special case of Proposition 3 in GW2.

It is tempting to conclude that $\Omega(1, \mu)$ or $\Omega(1, \mu)^{0}$ is $A_{\mathfrak{q}}(\lambda)$ as in Lemma 9.2.1. This is not the case as we will see in one special case below.

9.3. Quaternionic representations. We set $p=n=4$ and $m$ an even number greater than 4. We define $\lambda$ and $\mathfrak{q}$ as in Lemma 9.2.1. Using a standard spectral sequence argument, $\Omega(1)^{0}$ or $\Omega(1, \mu)^{0}$ embeds into $A^{\mathfrak{q}}(\lambda)$ and the image is the unique irreducible Harish-Chandra module generated by the lowest $K_{4, m}^{0}$-type.

In [GW1, Gross and Wallach show that the lowest $K_{4, m}^{0}$-type of $A^{\mathfrak{q}}(\lambda)$ generates the unique (proper) irreducible submodule which they denote by $\pi_{m-2}^{\prime}$. Hence we conclude that the irreducible submodule $\Omega(1)^{0}$ in the theta lift $\Omega(1)$ from $\widetilde{\mathrm{Sp}}(2, \mathbb{R})$ to $\tilde{\mathrm{O}}(4, m)$ is $\pi_{m-2}^{\prime}$ and it is a proper submodule in $A^{\mathfrak{q}}(\lambda)$.

9.4. The above discussion points to the following conjecture for $\mathfrak{s o}(n, r)$ in general.

Conjecture 9.4.1. In Lemma 9.2.1, $\Omega^{0}(1, \mu)$ is an irreducible submodule of $A^{\mathfrak{q}}(\lambda)$ generated by the lowest $K_{n, r}^{0}$-type. In particular, if $\lambda$ is in the weakly fair range, then $\Omega^{0}(1, \mu)$ is isomorphic to $A^{\mathfrak{q}}(\lambda)$.

When $\lambda$ lies in weakly fair range in the above conjecture, a related result of T. Kobayashi states that the corresponding $A^{\mathfrak{q}}(\lambda)$ is nonzero if and only if the following condition holds (see Theorem 3(5) and Eq. (2.6.3) in [Ko1]):

(T) $\mu_{p-r} \geq \frac{n-m}{2}$ if $p>r$. 
Furthermore if $A^{\mathfrak{q}}(\lambda) \neq 0$, then it is irreducible. On the other hand, one checks that for $\lambda$ in the weakly fair range, $\Omega^{0}(1, \mu) \neq 0$ if and only if Condition (T) holds.

The discussion in 9.3 shows that above conjecture is true if $n=4$. In this case Corollary 5.1.4 coincides with the restriction formula in [Lo]. A check against the list of $\tilde{\mathrm{O}}(n, 1)$ representations also shows that the conjecture is true if $r=1$.

9.5. We end our investigation of the dual pair $(\widetilde{\mathrm{Sp}}(n, \mathbb{R}), \tilde{\mathrm{O}}(n, m))$ with another conjecture. Suppose we replace $\tilde{\mathrm{O}}(n, r) \times_{2} \tilde{\mathrm{O}}(m-r)$ in the see-saw pair in (5) with $\tilde{\mathrm{O}}\left(n^{\prime}, m^{\prime}\right) \times_{2} \tilde{\mathrm{O}}\left(n^{\prime \prime}, m^{\prime \prime}\right)$ where $n=n^{\prime}+n^{\prime \prime}$ and $m=m^{\prime}+m^{\prime \prime}$. We assume that $p$ is very small compared to $n^{\prime}, n^{\prime \prime}, m^{\prime}$ and $m^{\prime \prime}$. Let $\mu$ be a genuine highest weight of $\tilde{\mathrm{O}}\left(r^{\prime}\right)$ and suppose $\Omega_{p}^{n, m}(1), \Omega_{p}^{n^{\prime}, m^{\prime}}(1, \mu)$ and $\Omega_{p}^{n^{\prime \prime}, m^{\prime \prime}}(1, \mu)$ are irreducible and unitarizable. Using the see-saw pair argument as in Lemma 4.2.1] there exists a nonzero $\left(\mathfrak{s o}\left(n^{\prime}, m^{\prime}\right) \times \mathfrak{s o}\left(n^{\prime \prime}, m^{\prime \prime}\right)\right)$-morphism

$$
\Omega_{p}^{n, m}(1) \rightarrow \Omega_{p}^{n^{\prime}, m^{\prime}}(1, \mu) \otimes \Omega_{p}^{n^{\prime \prime}, m^{\prime \prime}}(1, \mu) .
$$

Theorem $\mathrm{C}$ and $\S 9$ in $\mathrm{KoO}$ study the situation where $p=1$ and this motivates our next conjecture.

Conjecture 9.5.1. The tensor product $\Omega_{p}^{n^{\prime}, m^{\prime}}(1, \mu) \otimes \Omega_{p}^{n^{\prime \prime}, m^{\prime \prime}}(1, \mu)$ occurs discretely in the unitary representation whose Harish-Chandra module is $\Omega_{p}^{n, m}(1)$.

\section{Appendix A. The Howe quotient $\Omega_{p}^{n, n}(1)$ With Respect TO THE DUAL PAIR $(\widetilde{\operatorname{Sp}}(p, \mathbb{R}), \tilde{O}(n, n))$}

\section{by Soo Teck Lee}

A.1. In this appendix we shall study the structure of the Howe quotient $\Omega_{p}^{n, m}(1)$ in the case when $n=m$ by following the method of [LZ1] and [LZ2], that is, we embed $\Omega_{p}^{n, n}(1)$ into some degenerate principal series of $\mathrm{O}(n, n)$.

A.2. Representations of $\tilde{\mathrm{O}}(n)$. If $\lambda$ is given in (77) (resp. (8) ), we set $e(\lambda)=1$ (resp. $e(\lambda)=-1)$. We also denote $\lambda$ by $\left(\lambda^{\prime}, e(\lambda)\right)$ where $r=[n / 2]$ and $\lambda^{\prime}=$ $\left(a_{1}, \ldots, a_{k}, 0, \ldots, 0\right)_{r}$.

A.2.1. We first describe the structure of the degenerate principal series representation $I_{n, n}(s, \sigma)$ of $\tilde{\mathrm{O}}(n, n)$ in $\$ 5.4$. With some work, it can be deduced from a similar degenerate principal series of $\mathrm{SO}_{0}(n, n)$ whose structure is known ([]], $\mathrm{Sa}$, Zha]).

Let $r=[n / 2]$. Under the action of $\tilde{\mathrm{O}}(n) \times \tilde{\mathrm{O}}(n)$,

$$
I_{n, n}(s, \sigma)=\sum_{\lambda \in \Lambda^{+}(r), e= \pm 1} S(\lambda, e),
$$

where for each $\lambda \in \Lambda^{+}(r)$ and $e= \pm 1$,

$$
S(\lambda, e) \cong \tau_{n}^{(\lambda, e)} \otimes \tau_{n}^{\left(\lambda,(-1)^{\sigma} e\right) .}
$$


If $s \in \frac{n-1}{2}+\mathbb{Z}$, then let $t=t(s)=\max \left(0, \frac{n-1}{2}-|s|\right)$. For $t+1 \leq i \leq r$, we define the following subspace of $I_{n, n}(s, \sigma)$ :

$$
\begin{aligned}
R(n, s, \sigma, i)=\sum\left\{S(\lambda, e): \lambda_{i} \geq|s|-\frac{n-1}{2}+i \geq \lambda_{i+1},\right. \\
e=\left\{\begin{array}{ll}
1 & \text { if } n=2 r \text { is even and } \lambda_{r} \neq 0, \\
\pm 1 & \text { otherwise. }
\end{array}\right\} .
\end{aligned}
$$

If $|s| \leq \frac{n-1}{2}$ and $\varepsilon= \pm 1$, we also define

$$
R^{\varepsilon}(n, s, \sigma)=\sum\left\{S(\lambda, \varepsilon): \lambda_{t+1}=0\right\} .
$$

We refer to [LL1] for the definition of the module diagram of a representation of finite length. The following theorem summarizes the structure of $I_{n, n}(s, \sigma)$ :

Theorem A.2.1. Let $s \in \mathbb{C}$.

(a) If $s+\frac{n-1}{2} \notin \mathbb{Z}$, then $I_{n, n}(s, \sigma)$ is irreducible.

(b) Suppose that $s+\frac{n-1}{2} \in \mathbb{Z}$.

(i) If $s \leq-\frac{n+1}{2}$, then the module diagram of $I_{n, n}(s, \sigma)$ is given as follows:

$$
R(n, s, \sigma, r) \rightarrow R(n, s, \sigma, r-1) \rightarrow \cdots \rightarrow R(n, s, \sigma, 0) .
$$

(ii) If $-\frac{n-1}{2} \leq s \leq 0$, then the module diagram of $I_{n, n}(s, \sigma)$ is given as follows:

$$
R(n, s, \sigma, r) \rightarrow R(n, s, \sigma, r-1) \rightarrow \cdots \rightarrow R(n, s, \sigma, t+1) \searrow \begin{aligned}
& R^{+}(n, s, \sigma) \\
& R^{-}(n, s, \sigma),
\end{aligned}
$$

where $t=s+\frac{n-1}{2}$. In particular, if $n$ is odd and $s=0$, then

$$
I_{n, n}(0, \sigma)=R^{+}(n, 0, \sigma) \oplus R^{-}(n, 0, \sigma)
$$

is a sum of two irreducible submodules.

(iii) If $s>0$, then the diagram of $I_{n, n}(s, \sigma)$ can be obtained by reversing that of $I_{n, n}(-s, \sigma)$ given in (35) or (36).

(iv) $R^{ \pm}(n, s, \sigma)$ are unitarizable. $R(n, s, \sigma, i)$ is unitarizable iff $n$ is even and $i=r$.

A.2.2. By the work of [Zhu], there is an $\tilde{\mathrm{O}}(n, n)$-embedding

$$
\Omega_{p}^{n, n}(1) \stackrel{\psi}{\longleftrightarrow} I_{n, n}\left(p-\frac{n-1}{2}, 0\right)
$$

Now under the action of $\tilde{\mathrm{O}}(n) \times \tilde{\mathrm{O}}(n)$,

$$
\Omega_{p}^{n, n}(1)=\sum_{\lambda_{p+1}=0} \tau_{n}^{\lambda} \otimes \tau_{n}^{\lambda} .
$$

Thus by matching the $\tilde{\mathrm{O}}(n) \times \tilde{\mathrm{O}}(n)$ types and Theorem A.2.1, one immediately obtains the structure of $\Omega_{p}^{n, n}(1)$. The results are given in the following theorem.

Theorem A.2.2. Let $s=p-\frac{n-1}{2}$ and $r=\left[\frac{n}{2}\right]$.

(i) If $1 \leq p \leq \frac{n-1}{2}$, then

$$
\psi\left(\Omega_{p}^{n, n}(1)\right)=R^{+}(n, s, 0) .
$$

Thus in this case, $\Omega_{p}^{n, n}(1)$ is irreducible. 
(ii) If $\frac{n}{2} \leq p \leq n-1$, then $\psi\left(\Omega_{p}^{n, n}(1)\right)$ is the submodule of $I_{n, n}(s, 0)$ with the diagram

$$
R^{+}(n, s, 0) \rightarrow R(n, s, 0, n-p) \rightarrow \cdots \rightarrow R(n, s, 0, r) .
$$

(iii) If $p \geq n$, then

$$
\psi\left(\Omega_{p}^{n, n}(1)\right)=I_{n, n}(s, 0) .
$$

So the diagram of $\Omega_{p}^{n, n}(1)$ is given by

$\psi^{-1}(R(n, s, 0,0)) \rightarrow \psi^{-1}(R(n, s, 0,1)) \rightarrow \cdots \rightarrow \psi^{-1}(R(n, s, 0, r))$.

In particular, the theta lift $\psi^{-1}(R(n, s, 0,0))$ is finite-dimensional.

One interesting case is when $n=2 r=2 p$ is even, that is when the dual pair is $(\widetilde{\mathrm{Sp}}(p, \mathbb{R}), \tilde{\mathrm{O}}(2 p, 2 p))$. By the above theorem, the image of Howe quotient $\Omega_{p}^{2 p, 2 p}(1)$ in $I_{n, n}(1 / 2,0)$ has the diagram

$$
R^{+}(2 p, 1 / 2,0) \rightarrow R(2 p, 1 / 2,0, p),
$$

which is not irreducible. Also see Remark 5.3 in [Li1.

\section{REFERENCES}

[Ad] J. Adams, The Theta correspondences over $\mathbb{R}$ : Workshop at the University of Maryland. (1994).

[Al] J. Alperin, Diagram for modules, J. Pure Appl. Algebra 16 (1980), 111-119. MR0556154 (81h:16047)

[DER] M. Davidson, T. Enright, R. J. Stanke, Differential operators and highest weight representations. Mem. Amer. Math. Soc. 94 (1991). MR.1081660 (92c:22034)

[EHW] T. Enright, R. Howe and N. Wallach, A classification of unitary highest weight modules. Representation theory of reductive groups (Park City, Utah, 1982), 97-143, Progr. Math., 40, Birkhäuser Boston, Boston, MA, 1983. MR733809 (86c:22028)

[GZ] I. M. Gelfand and M. L. Tsetlin, Finite dimensional representations of the group of unimodular matrices, in "Gelfand, Izrail M. Collected Papers," Vol. II, 653-656, Springer-Verlag, Berlin, New York, 1988.

[GoW] Roe Goodman and Nolan R. Wallach, Representations and Invariants of the Classical Groups Cambridge Univ. Press, 1998; third corrected printing, 2003. MR1606831 (99b:20073)

[GW1] B. Gross and N. Wallach, On quaternionic discrete series representations, and their continuations. J. Reine Angew. Math. 481 (1996), 73-123. MR1421947(98f:22022)

[GW2] B. Gross and N. Wallach, Restriction of small discrete series representations to symmetric subgroups. The mathematical legacy of Harish-Chandra (Baltimore, MD, 1998), 255-272, Proc. Sympos. Pure Math., 68, AMS, Providence, RI, 2000. MR1767899 (2001f:22042)

[He1] Hongyu He, Theta correspondence. I. Semistable range: construction and irreducibility. Commun. Contemp. Math. 2 (2000), no. 2, 255-283. MR1759791 (2001f:22043)

[He2] Hongyu He, Compositions of theta correspondences. Adv. Math. 190 (2005), no. 2, 225263. MR2102656 (2005h:22021)

[Hi] T. Hirai, On infinitesimal operators of irreducible representations of the Lorentz group of $n$-th order. Proc. Japan Acad. 38 (1962) 83-87; On irreducible representations of the Lorentz group of $n$-th order. Proc. Japan Acad. 38 (1962) 258-262. MR0138703 (25:2146)

[Ho1] R. Howe, Remarks on classical invariant theory. Trans. Amer. Math. Soc. 313 (1989), no. 2, 539-570. MR0986027 (90h:22015a)

[Ho2] R. Howe Transcending classical invariant theory. J. Amer. Math. Soc. 2 no. 3 (1989). MR0985172 (90k:22016)

[Ho3] R. Howe Perspectives on invariant theory: Schur duality, multiplicity-free actions and beyond. The Schur lectures (1992) (Tel Aviv), 1-182, Israel Math. Conf. Proc., 8, Bar-Ilan Univ., Ramat Gan, 1995. MR.1321638 (96e:13006)

[HL] J-S. Huang and J-S. Li, Unipotent representations attached to spherical nilpotent orbits. Amer. J. Math. 121 (1999), no. 3, 497-517. MR1738410 (2000m:22018) 
[HT] R. Howe and E. Tan, Homogeneous functions on light cones: the infinitesimal structure of some degenerate principal series representations, Bull. Amer. Math. Soc. 28 (1993) 1-74. MR $1172839(93 \mathrm{j}: 22027)$

[J] K. Johnson, Degenerate principal series and compact groups, Math. Ann. 287 (1990), 703-718. MR 1066825 (91h:22028)

[Ko1] T. Kobayashi, Singular Unitary Representations and discrete series for indefinite Stiefel manifolds $\mathrm{U}(p, q ; \mathbb{F}) / \mathrm{U}(p-m, q ; \mathbb{F})$. Mem. Amer. Math. Soc., 95, no. 462 (1992). MR 1098380 (92f:22023)

[Ko2] T. Kobayashi, Discrete decomposability of the restriction of $A_{\mathfrak{q}}(\lambda)$ with respect to reductive subgroups. III. Restriction of Harish-Chandra modules and associated varieties. Invent. Math. 131 (1998), no. 2, 229-256. MR1608642 (99k:22021)

[KG] A. U. Klimyk and A. M. Gavrilik, The representations of the groups $\mathrm{U}(n, 1)$ and $\mathrm{SO}_{0}(n, 1)$, preprint ITP-76-39E, Instituent for Theoretical Physics Kiev, USSR,1976.

[KoO] T. Kobayashi and B. Orsted, Analysis of the minimal representation of $\mathrm{O}(p, q) I$, II, III. Adv. in Math 180 (2003) 486-512, 513-550, 551-595. MR2020550 (2004k:22018a) MR.2020551 (2004k:22018b) MR2020552 (2004k:22018c)

[KR] S. Kudla and S. Rallis, Degenerate principal series and invariant distributions, Isreal Journal of Math. 69 (1990), 25-45. MR1046171 (91e:22016)

[KV] M. Kashiwara and M. Vergne On the Segal-Shale-Weil representations and harmonic polynomials. Invent. Math. 44 (1978), no. 1, 1-47. MR0463359 (57:3311)

$[\mathrm{KnV}]$ A. W. Knapp and D.A. Vogan Cohomological induction and unitary representations. Princeton Mathematical Series, 45. Princeton University Press, Princeton, NJ, 1995. MR $1330919(96 \mathrm{c}: 22023)$

[Le] S. T. Lee, On some degenerate principal series representations of $\mathrm{U}(n, n)$. J. Funct. Anal. 126 (1994), 305-366. MR1305072 (95j:22023)

[LL1] S. T. Lee and H. Y. Loke, Degenerate principal series representation of $\mathrm{U}_{p, q}$ and $\operatorname{Spin}_{0}(p, q)$. Comp. Math. 132 (2002), 311-348. MR.1918135 (2003g:22014)

[LL2] S. T. Lee and H. Y. Loke, Degenerate principal series representation of $\operatorname{Sp}(p, q)$. Israel Journal of Mathematics 137 (2003), 355-379. MR2013362 (2004m:22022)

[LZ1] S. T. Lee and C.-B. Zhu, Degenerate principal series and local theta correspondence. Trans. AMS 350 No. 12 (1998), 5017-5046. MR1443883 (99c:22021)

[LZ2] S. T. Lee and C.-B. Zhu, Degenerate principal series and local theta correspondence. II. Israel J. Math. 100 (1997), 29-59. MR1469104 (99c:22022)

[Li1] J.-S. Li, Singular unitary representations of classical groups, Invent. Math. 97 (1990), 237-255. MR.1001840 (90h:22021)

[Li2] J.-S. Li, Theta lifting for unitary representations with nonzero cohomology, Duke Math. J. 61 (1990), no. 3, 913-937. MR1084465 (92f:22024)

[Li3] J.-S. Li, Degenerate principal series of classical groups: the phenomenon of long complementary series, preprint.

[Lo] H. Y. Loke, Restrictions of quaternionic representations. J. Funct. Anal. 172 (2000), no. 2, 377-403. MR1753179 (2001i:22017)

[NZ1] K. Nishiyama and C.-B. Zhu, Theta lifting of holomorphic discrete series: The case of $\mathrm{U}(p, q) \times \mathrm{U}(n, n)$. Trans. Amer. Math. Soc. 353 (2001), 3327-3345. MR1828608 (2002e:22017)

[NZ2] K. Nishiyama and C.-B. Zhu, Theta lifting of unitary lowest weight modules and their associated cycles. Duke Mathematical Journal 125, No. 3 (2004), 415-465. MR2166751

$[\mathrm{PT}] \quad$ A. Paul and P. Trapa, One-dimensional representations of $\widetilde{\mathrm{U}}(p, q)$ and the Howe correspondence. J. Funct. Anal. 195 (2002), 129-166. MR1934355(2004a:22014)

[Pr] R. Proctor, Young tableaux, Gelfand patterns, and branching rules for classical groups. J. Algebra 164 (1994), no. 2, 299-360. MR1271242 (96e:05180)

[Pz] T. Przebinda, The duality correspondence of infinitesimal characters. Colloq. Math. 70. (1996), no. 1, 93-102. MR.1373285 (96m:22034)

[Sa] S. Sahi, Unitary representations on the Shilov boundary of a symmetric tube domain, in "Representation theory of Groups and Algebras," Contemp. Math. 145 (1993), 275-286, Amer. Math. Soc., Providence. MR1216195 (94e:22029)

[Tan] E.-C Tan, On the theta lift for the trivial representation. Analysis on homogeneous spaces and representation theory of Lie groups, Okayama-Kyoto (1997), 213-234, Adv. Stud. Pure Math., 26, Math. Soc. Japan, Tokyo, 2000. MR1770722 (2001f:22049) 
[TZ] E.-C Tan and C.-B. Zhu, On certain distinguished unitary representations supported on null cones. Am. J. Math. 120 (1997), 1059-1076. MR.1646054 (99h:22019)

[VK] N. Ja. Vilenkin and A. U. Klimyk, Representations of Lie groups and special functions, Vol 3: Classical and Quantum groups and special functions, Kluwer Academic Publishers, (1992). MR1206906 (96f:33043)

[Vo1] D. Vogan, The algebraic structure of the representation of semisimple Lie groups. I. Ann. of Math. (2) 109 (1979), no. 1, 1-60. MR0519352 (81j:22020)

[Vo2] D. Vogan, Representations of real reductive Lie groups, Progress in Math. 15, Birkhäuser, Boston, MA, 1981. MR0632407 (83c:22022)

[Vo3] D. Vogan, Unitarizability of certain series of representations. Ann. of Math. 120 (1984), 141-187. MR0750719 (86h:22028)

[Vo4] D. Vogan, Associated varieties and unipotent representations. Harmonic analysis on reductive groups (Brunswick, ME, 1989), 315-388, Progr. Math., 101, Birkhäuser Boston, Boston, MA, 1991. MR1168491 (93k:22012)

[VZ] D. A. Vogan and G. J. Zuckerman, Unitary representations with nonzero cohomology. Compositio Math. 53 (1984), no. 1, 51-90. MR0762307 (86k:22040)

[Zha] G. Zhang, Jordan algebras and generalized principal series representation, Math. Ann. 302 (1995), 773-786. MR:1343649 (97h:22012)

[Zhu] C.-B. Zhu, Invariant distributions of classical groups. Duke Math. J. 65 (1992), 85-119. MR 1148986 (92k:22022)

[ZH] C.-B. Zhu and J.-S. Huang, On certain small representations of indefinite orthogonal groups. Representation Theory 1, (1997), 190-206 (electronic). MR1457244 (98j:22026)

Department of Mathematics, National University of Singapore, 2, Science Drive, SingAPORE 117543

E-mail address: matlhy@nus.edu.sg

Department of Mathematics, National University of Singapore, 2, Science Drive, SingAPORE 117543

E-mail address: matleest@nus.edu.sg 